\title{
Modeling of spiral wound pervaporation modules with application to the separation of styrene/ethylbenzene mixtures
}

\author{
Bing Cao, Michael A. Henson* \\ Department of Chemical Engineering, Louisiana State University, Baton Rouge, LA 70803-7303, USA
}

Received 25 April 2001; received in revised form 6 August 2001; accepted 9 August 2001

\begin{abstract}
A mathematical model is derived to simulate the performance of spiral wound membrane modules for the pervaporative separation of binary liquid mixtures. Permeation through the polymer membrane is described by a detailed solution-diffusion model. Flory-Huggins theory is used to predict solubility of the penetrants, while the composition and temperature dependence of the diffusion coefficients are described by phenomenological relations. Unknown parameters of the solution-diffusion model are determined from sorption and flux data using nonlinear least-squares estimation. Standard correlations are used to estimate the mass transfer resistance due to the liquid boundary layer on the feed side of the module. The solution-diffusion model is coupled to differential mass, momentum and energy balances on the feed and permeate sides of the module to predict separation performance. The resulting model is two-dimensional as the feed-side and permeate-side stream properties vary in both the feed and permeate flow directions. Required input data to the model includes the feed flow rate, composition and temperature, the outlet permeate pressure, the membrane properties and the module dimensions. A numerical solution technique based on the use of the shooting method in the permeate flow direction and numerical integration in the feed flow direction is proposed. The model yields predictions of the feed-side and permeate-side stream properties as a function of both spatial coordinates. The separation of styrene and ethylbenzene with a polyurethane membrane is used to illustrate the modeling approach. A single module and a 10-module system with interstage heating are simulated to demonstrate potential uses of the pervaporation model. ( 2002 Elsevier Science B.V. All rights reserved.
\end{abstract}

Keywords: Pervaporation; Membrane modules; Modeling; Parameter estimation; Simulation

\section{Introduction}

The separation of styrene/ethylbenzene mixtures is a problem of considerable industrial importance. Styrene is an important petrochemical product and is used extensively in the manufacturing of plastic materials. The dehydrogenation of ethylbenzene is the dominant technology for styrene production [24]. An upper limit on the reaction temperature must be en-

\footnotetext{
* Corresponding author. Tel.: +1-225-578-3690;

fax: $+1-225-388-1476$.

E-mail address: henson@che.lsu.edu (M.A. Henson).
}

forced to avoid polymerization and thermal cracking of the styrene. As a result, the ethylbenzene conversion is approximately $75 \%$ and the reactor effluent contains a significant amount of ethylbenzene in addition to the styrene product. The effluent stream is separated to produce ethylbenzene for recycle to the reactor and to remove undesirable reaction byproducts. The styrene/ethylbenzene separation currently is performed via conventional distillation [24]. Because the boiling points of styrene and ethylbenzene are very similar, a distillation column with as many as 100 theoretical stages and a high reflux ratio is required to achieve the desired styrene purity of 


\begin{tabular}{|c|c|}
\hline \multicolumn{2}{|c|}{ Nomenclature } \\
\hline$a$ & activity \\
\hline$a_{1}-a_{3}$ & solution model parameters \\
\hline$A$ & constant in the Margules equation \\
\hline$A_{11}-A_{22}$ & diffusion coefficient model parameters \\
\hline$b_{1}-b_{3}$ & solution model parameters \\
\hline$C$ & mass concentration $\left(\mathrm{g} / \mathrm{cm}^{3}\right)$ \\
\hline$C_{p}$ & $\begin{array}{l}\text { heat capacity at constant pressure } \\
(\mathrm{J} / \mathrm{g} \mathrm{K})\end{array}$ \\
\hline$d$ & flow channel width $(\mathrm{cm})$ \\
\hline$D$ & diffusion coefficient $\left(\mathrm{cm}^{2} / \mathrm{s}\right)$ \\
\hline$D^{0}$ & $\begin{array}{l}\text { diffusion coefficient at infinite dilution } \\
\left(\mathrm{cm}^{2} / \mathrm{s}\right)\end{array}$ \\
\hline$D_{12}^{1}$ & $\begin{array}{l}\text { diffusion coefficient of liquid } \\
\text { phase components }\left(\mathrm{cm}^{2} / \mathrm{s}\right)\end{array}$ \\
\hline$f$ & Fanning friction factor \\
\hline$F$ & mass flow rate $(\mathrm{g} / \mathrm{s})$ \\
\hline$G^{\mathrm{E}}$ & excess Gibbs free energy $(\mathrm{J} / \mathrm{mol})$ \\
\hline$\Delta G_{\text {mix }}$ & Gibbs free energy of mixing $(\mathrm{J} / \mathrm{mol})$ \\
\hline$H$ & membrane element height $(\mathrm{cm})$ \\
\hline$J$ & mass flux $\left(\mathrm{g} / \mathrm{cm}^{2} \mathrm{~s}\right)$ \\
\hline$\tilde{J}$ & molar flux $\left(\mathrm{mol} / \mathrm{cm}^{2} \mathrm{~s}\right)$ \\
\hline$J^{*}$ & $\begin{array}{l}\text { flux at zero permeate pressure } \\
\left(\mathrm{g} / \mathrm{cm}^{2} \mathrm{~s}\right)\end{array}$ \\
\hline$k$ & mass transfer coefficient $(\mathrm{s} / \mathrm{cm})$ \\
\hline$l$ & membrane thickness $(\mu \mathrm{m})$ \\
\hline$L$ & membrane element length $(\mathrm{cm})$ \\
\hline$m$ & permeate-side molar flux $\left(\mathrm{mol} / \mathrm{cm}^{2} \mathrm{~s}\right)$ \\
\hline$M$ & $\begin{array}{l}\text { molecular weight }(\mathrm{g} / \mathrm{mol}) \text {, number of } \\
\text { quadrature points }\end{array}$ \\
\hline$M_{\mathrm{c}}$ & $\begin{array}{l}\text { molecular weight between two } \\
\text { crosslinks of the polymer } \\
\text { membrane }(\mathrm{g} / \mathrm{mol})\end{array}$ \\
\hline$n$ & $\begin{array}{l}\text { mole fraction, feed-side molar flux } \\
\left(\mathrm{mol} / \mathrm{cm}^{2} \mathrm{~s}\right) \text {, number of grid points }\end{array}$ \\
\hline$P$ & permeate-side pressure $(\mathrm{Pa})$ \\
\hline$P_{H}$ & $\begin{array}{l}\text { permeate-side pressure at the } \\
\text { collection tube }(\mathrm{Pa})\end{array}$ \\
\hline$P^{\mathrm{sat}}$ & saturation vapor pressure $(\mathrm{Pa})$ \\
\hline$Q$ & feed-side volumetric flow rate $\left(\mathrm{cm}^{3} / \mathrm{s}\right)$ \\
\hline$R$ & gas constant $(\mathrm{J} / \mathrm{mol} \mathrm{K})$ \\
\hline $\operatorname{Re}$ & Reynolds number \\
\hline$S$ & $\begin{array}{l}\text { solubility of pure component in the } \\
\text { polymer membrane }\left(\mathrm{g} / \mathrm{cm}^{3} \text { of polymer) }\right.\end{array}$ \\
\hline$S c$ & Schmidt number \\
\hline$S h$ & local Sherwood number \\
\hline
\end{tabular}

\begin{tabular}{|c|c|}
\hline$S h_{\mathrm{L}}$ & average Sherwood number \\
\hline$T$ & feed-side temperature $(\mathrm{K})$ \\
\hline$u$ & $\begin{array}{l}\text { permeate-side velocity }(\mathrm{cm} / \mathrm{s}) \text {, volume } \\
\text { fraction in the polymer membrane on } \\
\text { a polymer free basis }\end{array}$ \\
\hline$v$ & feed-side velocity $(\mathrm{cm} / \mathrm{s})$ \\
\hline$V$ & $\begin{array}{l}\text { molar volume }\left(\mathrm{cm}^{3} / \mathrm{mol}\right) \text {, specific } \\
\text { volume }\left(\mathrm{cm}^{3} / \mathrm{g}\right)\end{array}$ \\
\hline$w$ & quadrature weight \\
\hline$W$ & membrane element width $(\mathrm{cm})$ \\
\hline$x$ & $\begin{array}{l}\text { mole fraction in liquid mixture, } \\
\text { membrane leaf length variable }(\mathrm{cm})\end{array}$ \\
\hline$\Delta x$ & discretized length element $(\mathrm{cm})$ \\
\hline$y$ & $\begin{array}{l}\text { mole fraction in vapor mixture, } \\
\text { membrane leaf height variable }(\mathrm{cm})\end{array}$ \\
\hline$\Delta y$ & discretized height element $(\mathrm{cm})$ \\
\hline$z$ & membrane leaf width variable $(\mathrm{cm})$ \\
\hline \multicolumn{2}{|c|}{ Greek letters } \\
\hline$\alpha$ & selectivity \\
\hline$\beta$ & $\begin{array}{l}\text { update constant for shooting } \\
\text { calculations }\end{array}$ \\
\hline$\chi$ & Flory-Huggins interaction parameter \\
\hline$\epsilon$ & tolerance for shooting calculations \\
\hline$\phi$ & volume fraction in membrane \\
\hline$\phi^{0}$ & volume fraction at membrane surface \\
\hline$\gamma$ & activity coefficient \\
\hline$\varphi$ & stage cut \\
\hline$\lambda$ & latent heat of vaporization $(\mathrm{J} / \mathrm{g})$ \\
\hline$\mu$ & viscosity (cP) \\
\hline$\theta$ & unknown parameters vector \\
\hline$\rho$ & mass density $\left(\mathrm{g} / \mathrm{cm}^{3}\right)$ \\
\hline$\tilde{\rho}$ & molar density $\left(\mathrm{mol} / \mathrm{cm}^{3}\right)$ \\
\hline$\xi$ & quadrature point \\
\hline$\Psi$ & volume fraction in liquid mixture \\
\hline \multicolumn{2}{|c|}{ Subscripts } \\
\hline $\mathrm{f}$ & feed \\
\hline 1 & liquid property \\
\hline $\mathrm{m}$ & membrane property \\
\hline $\mathrm{p}$ & $\begin{array}{l}\text { polymer property, local permeate } \\
\text { property }\end{array}$ \\
\hline $\mathrm{t}$ & total property \\
\hline $\mathrm{v}$ & vapor property \\
\hline$x$ & $x$-component \\
\hline$z$ & $z$-component \\
\hline 0 & reference \\
\hline
\end{tabular}




\begin{tabular}{|c|c|}
\hline 1 & $\begin{array}{l}\text { liquid component in binary and } \\
\text { ternary mixtures }\end{array}$ \\
\hline 2 & $\begin{array}{l}\text { polymer in binary mixture and liquid } \\
\text { component in ternary mixture }\end{array}$ \\
\hline 3 & polymer in ternary mixture \\
\hline \multicolumn{2}{|c|}{ Superscripts } \\
\hline $\mathrm{b}$ & binary property of the ternary mixture \\
\hline $\mathrm{b} i$ & $\begin{array}{l}\text { binary property between liquid component } \\
i \text { and the polymer in the ternary mixture }\end{array}$ \\
\hline L & liquid property \\
\hline $\mathrm{P}$ & polymer property \\
\hline sat & property of the saturated vapor \\
\hline V & vapor property \\
\hline * & average property, dimensionless variable \\
\hline
\end{tabular}

99.9\%. The distillation process is very capital and energy intensive, so there is considerable motivation to develop simpler and more cost effective separation technologies.

Pervaporation has been proposed as an economically viable technology for the separation of azeotropic and close-boiling mixture that pose difficulties for conventional distillation [11]. It has been estimated that the development of a high performance styrene/ethylbenzene pervaporation membrane could produce energy savings as large as $84 \%$ compared to distillation [14]. A poly(hexamethylene sebacate) (PHS)-based polyurethane membrane for pervaporative separation of styrene/ethylbenzene mixtures has been synthesized and analyzed by the first author [4,5]. While the polyurethane membrane cannot be characterized as a high performance material according to the specifications in [14], the styrene permselectivity is sufficient for bulk separation. Consequently, the membrane could be used to develop a hybrid pervaporation-distillation process that offers the potential for lower capitals costs and better energy efficiency than conventional distillation.

To realize such a hybrid process, it is necessary to construct a membrane module that provides adequate flux of the styrene enriched permeate stream. Many industrial pervaporation units consist of stainless steel plate and frame modules [9]. This configuration is relatively expensive compared to other types of membrane modules due to the low separation area to volume ratios achievable. Commercial spiral wound modules have been developed for some solvent dehydration applications $[9,22]$, but to our knowledge spiral wound modules for bulk separation of hydrocarbons are not available. The polyurethane membrane investigated in $[4,5]$ appears to have the necessary material properties to construct a spiral wound module. In this paper, the spiral wound configuration is assumed for derivation of the pervaporation model. Indeed, it is likely that such a module configuration would be required for styrene/ethylbenzene separations due to the large flow rates involved.

Transport models of pervaporation modules have been proposed by several investigators. Rautenbach and Albrecht $[20,21]$ derive transport equations for a hollow fiber module to study the pervaporative separation of benzene and cyclohexane. The permeation behavior is described by a solution-diffusion model similar to that presented in Section 2.1. However, the other transport equations are inappropriate for spiral wound modules where the feed-side and permeate-side flow directions are perpendicular and the problem is two-dimensional. Hickey and Gooding [13] develop a transport model to evaluate the performance of spiral wound modules for pervaporative removal of volatile organic compounds (VOCs) from water. Because VOCs typically are present in small concentrations, a very simple flux model based on Raoult's law for water and Henry's law for the VOCs is proposed. These assumptions are invalid for bulk hydrocarbon separations where both components are present in appreciable amounts and the mass transfer coefficients are concentration and temperature-dependent. The model in [13] consists of permeate-side mass and momentum balances as well as a feed-side VOC balance. The momentum balance is crucial because permeate pressure buildup has a strong effect on the mass transfer driving force. By introducing several simplifying assumptions, the transport equations are reduced to a set of nonlinear ordinary differential equations with mixed boundary conditions. However, the assumptions of constant feed-side flow rate and isothermal operation are invalid for hydrocarbon separations.

In this paper, a transport model for spiral wound pervaporation modules is derived by coupling a detailed solution-diffusion model to differential mass, momentum and energy balances on the feed and permeate sides of the module. We believe this is the 
most detailed pervaporation model available in the open literature for describing bulk separation of binary mixtures with solution-diffusion membranes. The model consists of coupled nonlinear ordinary differential equations with two spatial coordinates and mixed boundary conditions. Efficient and reliable model solution is a non-trivial problem that motivates the development of specialized numerical techniques that exploit the unique structure of the model.

The remainder of the paper is organized as follows. In Section 2, the solution-diffusion model is described and a nonlinear least-squares estimation technique for determining the model parameters from sorption and flux data is presented. The solution-diffusion model is evaluated for the separation of styrene and ethylbenzene with a polyurethane membrane. Differential mass, momentum and energy balances for the feed and permeate sides of the module are derived in Section 3. An iterative algorithm to solve the resulting nonlinear differential equation model based on the use of the shooting method in the permeate flow direction and numerical integration in the feed flow direction is proposed. In Section 4, the pervaporative separation of styrene/ethylbenzene mixtures is used to illustrate the modeling approach. A 10-module system with interstage heating is simulated to demonstrate the potential for hybrid pervaporation-distillation process development.

\section{Solution-diffusion model}

The solution-diffusion model is the accepted mechanism for describing permeation in polymer membranes [15,25]. According to this mechanism, pervaporation involves the following three steps: (i) the liquid species are dissolved into the membrane surface; (ii) the species diffuse through the membrane; (iii) the species desorb from the downstream membrane surface in the vapor phase. The solution and diffusion models used in this paper are presented in Sections 2.1 and 2.2, respectively. Also discussed in these subsections is the estimation of the solution-diffusion model parameters from sorption and flux data. A simple correlation model for capturing the effect of the feed-side liquid boundary layer resistance on the overall mass transfer coefficient is described in Section 2.3 .

\subsection{Solution model}

\subsubsection{Theory}

The component volume fractions sorbed into the membrane are predicted from thermodynamic properties of the liquid-polymer mixture. The solution model is based on Flory-Huggins theory [12] and utilizes the interaction parameter equations proposed by Mulder et al. [16]. When a polymer film is exposed to a pure liquid, Flory-Huggins theory yields the following relation for the pure component activity $a^{\mathrm{P}}$ :

$\ln a^{\mathrm{P}}=\ln \left(1-\phi_{2}\right)+\left(1-\frac{V_{1}}{V_{2}}\right) \phi_{2}+\chi \phi_{2}^{2}$

where $\phi_{2}$ is the volume fraction of the polymer, $V_{1}$ and $V_{2}$ the molar volumes of the liquid and polymer, respectively, and $\chi$ is the Flory-Huggins interaction parameter. The Gibbs free energy of mixing $\Delta G_{\text {mix }}$ of a ternary system comprised of a binary liquid mixture and a polymer membrane can be expressed as

$$
\begin{aligned}
\frac{\Delta G_{\text {mix }}}{R T}= & n_{1} \ln \phi_{1}+n_{2} \ln \phi_{2}+n_{3} \ln \phi_{3}+\chi_{12} n_{1} \phi_{2} \\
& +\chi_{13} n_{1} \phi_{3}+\chi_{23} n_{2} \phi_{3}
\end{aligned}
$$

where the subscripts 1 and 2 denote the liquid components and the subscript 3 denotes the polymer, $n_{i}$ and $\phi_{i}$ the mole fraction and volume fraction, respectively, of component $i, \chi_{i j}$ the interaction parameter between components $i$ and $j, T$ the temperature, and $R$ is the gas constant. The volume fraction $\phi_{i}$ is defined as

$\phi_{i}=\frac{n_{i} V_{i}}{\sum_{i=1}^{3} n_{i} V_{i}}$

As discussed below, the interaction parameters $\chi_{i j}$ are allowed to be concentration-dependent.

The interaction parameter $\chi_{12}$ between the two liquid components can be calculated using excess functions. The following equation holds for binary mixtures [18]:

$\frac{\Delta G_{\mathrm{mix}}}{R T}=x_{1} \ln x_{1}+x_{2} \ln x_{2}+\frac{G^{\mathrm{E}}}{R T}$

where $G^{\mathrm{E}}$ is the excess Gibbs free energy, and $x_{i}$ is the mole fraction of component $i$ in the liquid phase. The free energy of mixing of the binary 
mixture can be calculated using Flory-Huggins theory [12]:

$$
\begin{aligned}
\frac{\Delta G_{\text {mix }}}{R T}= & x_{1} \ln \Psi_{1}+x_{2} \ln \Psi_{2} \\
& +\chi_{12} \Psi_{1} \Psi_{2}\left(x_{1}+x_{2} \frac{V_{2}}{V_{1}}\right)
\end{aligned}
$$

where $\Psi_{i}$ is the volume fraction of component $i$ in the liquid phase:

$\Psi_{1}=\frac{V_{1} x_{1}}{V_{1} x_{1}+V_{2} x_{2}}, \quad \Psi_{2}=\frac{V_{2} x_{2}}{V_{1} x_{1}+V_{2} x_{2}}$

The excess Gibbs free energy can be expressed as

$\frac{G^{\mathrm{E}}}{R T}=x_{1} \ln \gamma_{1}^{\mathrm{L}}+x_{2} \ln \gamma_{2}^{\mathrm{L}}$

where $\gamma_{i}^{\mathrm{L}}=a_{i}^{\mathrm{L}} / x_{i}$ is the activity coefficient of component $i$ in the liquid phase, and $a_{i}^{\mathrm{L}}$ is the activity of component $i$ in the liquid phase. The liquid phase activities are estimated from vapor-liquid equilibrium data using the two-suffix Margules equation [18]:

$R T \ln \gamma_{1}^{\mathrm{L}}=A x_{2}^{2}, \quad R T \ln \gamma_{2}^{\mathrm{L}}=A x_{1}^{2}$

The Margules constant $A$ is determined from vapor-liquid equilibrium data using the following equation:

$$
\begin{aligned}
P^{\mathrm{sat}} & =\sum_{i=1}^{2} x_{i} \gamma_{i}^{\mathrm{L}} P_{i}^{\mathrm{sat}} \\
& =x_{1} P_{1}^{\mathrm{sat}} \exp \left[\frac{A}{R T} x_{2}^{2}\right]+x_{2} P_{2}^{\mathrm{sat}} \exp \left[\frac{A}{R T} x_{1}^{2}\right]
\end{aligned}
$$

where $P^{\text {sat }}$ is the vapor pressure of the binary mixture, and $P_{i}^{\text {sat }}$ is the temperature-dependent vapor pressure of pure component $i$. The following equation for $\chi_{12}$ is readily derived by combining Eqs. (3)-(5):

$\chi_{12}=\frac{1}{x_{1} \Psi_{2}}\left[x_{1} \ln \left(\frac{x_{1}}{\Psi_{1}}\right)+x_{2} \ln \left(\frac{x_{2}}{\Psi_{2}}\right)+\frac{A x_{1} x_{2}}{R T}\right]$

The interaction parameters $\chi_{13}$ and $\chi_{23}$ between the liquid components and the polymer in the ternary mixture can be determined from solubilities of the pure liquids in the polymer. From Eq. (1) it follows that the activity of pure component $i$ in the polymer is

$$
\ln a_{i}^{\mathrm{P}}=\ln \left(1-\phi_{3}^{\mathrm{b} i}\right)+\left(1-\frac{V_{i}}{V_{3}}\right) \phi_{3}^{\mathrm{b} i}+\chi_{i 3}^{\mathrm{b}}\left(\phi_{3}^{\mathrm{b} i}\right)^{2}
$$

where the superscript ' $b$ ' denotes a binary property of the ternary mixture, and the superscript ' $b i$ ' denotes a binary property between liquid component $i$ and the polymer in the ternary mixture. Equilibrium between the polymer phase and the pure liquid phase requires that

$a_{i}^{\mathrm{P}}=a_{i}^{\mathrm{L}}=1$

The following equation for the binary interaction parameter $\chi_{i 3}^{\mathrm{b}}$ is obtained from Eq. (8):

$\chi_{i 3}^{\mathrm{b}}=-\frac{\ln \left(1-\phi_{3}^{\mathrm{b} i}\right)-\left(1-\left(V_{i} / V_{3}\right)\right) \phi_{3}^{\mathrm{b} i}}{\left(\phi_{3}^{\mathrm{b} i}\right)^{2}}$

The polymer volume fraction $\phi_{3}^{\mathrm{b} i}$ for a binary mixture of polymer and liquid component $i$ is calculated as

$\phi_{3}^{\mathrm{b} i}=\frac{1}{\left(S_{i}^{\mathrm{b}} / \rho_{i}\right)+1}$

where $S_{i}^{\mathrm{b}}$ is the solubility of pure component $i$ in the polymer, and $\rho_{i}$ is the density of component $i$. Typically the ternary interaction parameters $\chi_{i 3}$ depend on the component concentrations in the polymer. They are calculated from the binary interaction parameter $\chi_{i 3}^{\mathrm{b}}$ using the empirical relation proposed in [16]:

$\chi_{13}=\chi_{13}^{\mathrm{b}}+a_{1} u_{2}^{2}+a_{2} u_{2}+a_{3}\left(\phi_{3}-\phi_{3}^{\mathrm{b} 1}\right)$

$\chi_{23}=\chi_{23}^{\mathrm{b}}+b_{1} u_{1}^{2}+b_{2} u_{1}+b_{3}\left(\phi_{3}-\phi_{3}^{\mathrm{b} 2}\right)$

where $u_{i}$ is the volume fraction of component $i$ in the polymer on a polymer free basis:

$u_{i}=\frac{\phi_{i}}{\phi_{1}+\phi_{2}}$

As discussed below, the constant parameters $a_{j}$ and $b_{j}$ can be estimated from solubility data for the ternary mixture.

The relation (1) can be extended to the ternary system that results when a polymer is exposed to a binary 
liquid mixture [17]:

$$
\begin{aligned}
\ln a_{1}^{\mathrm{P}}= & \ln \phi_{1}+\phi_{2}+\phi_{3}-\phi_{2} \frac{V_{1}}{V_{2}}-\phi_{3} \frac{V_{1}}{V_{3}} \\
& +\chi_{12} \phi_{2}\left(\phi_{2}+\phi_{3}\right)+\chi_{13} \phi_{3}\left(\phi_{2}+\phi_{3}\right) \\
& -\chi_{23} \phi_{2} \phi_{3} \frac{V_{1}}{V_{2}}-u_{1} u_{2} \phi_{2} \frac{\partial \chi_{12}}{\partial u_{2}} \\
& -u_{1} u_{2} \phi_{3} \frac{\partial \chi_{13}}{\partial u_{2}}-\phi_{1} \phi_{3}^{2} \frac{\partial \chi_{13}}{\partial \phi_{3}} \\
& +\frac{V_{1}}{V_{2}} u_{2}^{2} \phi_{3} \frac{\partial \chi_{23}}{\partial u_{1}}-\frac{V_{1}}{V_{2}} \phi_{2} \phi_{3}^{2} \frac{\partial \chi_{23}}{\partial \phi_{3}} \\
& +\frac{V_{1} \rho_{3}}{M_{\mathrm{c}}}\left(1-\frac{2 M_{\mathrm{c}}}{M}\right)\left(\phi_{3}^{1 / 3}-\frac{1}{2} \phi_{3}\right) \\
\ln a_{2}^{\mathrm{P}}= & \ln \phi_{2}+\phi_{1}+\phi_{3}-\phi_{1} \frac{V_{2}}{V_{1}}-\phi_{3} \frac{V_{2}}{V_{3}} \\
& +\chi_{12} \phi_{1} \frac{V_{2}}{V_{1}}\left(\phi_{1}+\phi_{3}\right)+\chi_{23} \phi_{3}\left(\phi_{1}+\phi_{3}\right) \\
& -\chi_{13} \phi_{1} \phi_{3} \frac{V_{2}}{V_{1}}-\frac{V_{2}}{V_{1}} u_{1}^{2} \phi_{2} \frac{\partial \chi_{12}}{\partial u_{2}} \\
& +\frac{V_{2}}{V_{1}} u_{1}^{2} \phi_{3} \frac{\partial \chi_{13}}{\partial u_{2}}-\frac{V_{2}}{V_{1}} \phi_{1} \phi_{3}^{2} \frac{\partial \chi_{13}}{\partial \phi_{3}} \\
& -u_{1} u_{2} \phi_{3} \frac{\partial \chi_{23}}{\partial u_{1}}-\phi_{2} \phi_{3}^{2} \frac{\partial \chi_{23}}{\partial \phi_{3}} \\
& +\frac{V_{2} \rho_{3}}{M_{\mathrm{c}}}\left(1-\frac{2 M_{\mathrm{c}}}{M_{3}}\right)\left(\phi_{3}^{1 / 3}-\frac{1}{2} \phi_{3}\right)
\end{aligned}
$$

where $\rho_{3}$ is the density of the polymer, $M_{3}$ the molecular weight of the polymer, and $M_{\mathrm{c}}$ is the molecular weight between two crosslinks of the polymer. For the PHS-based polyurethane membrane discussed previously, $M_{\mathrm{c}}$ is taken as the molecular weight of PHS and $M_{3}$ is assumed to be very large compared to $M_{\mathrm{c}}$. Equilibrium between the polymer phase and the binary liquid phase requires that

$a_{1}^{\mathrm{L}}=a_{1}^{\mathrm{P}}, \quad a_{2}^{\mathrm{L}}=a_{2}^{\mathrm{P}}$

As shown below, Eqs. (12)-(14) allow the volume fractions $\phi_{i}$ to be computed.

\subsubsection{Estimation of the solution model parameters}

Solubilities of the two liquid components in the polymer are obtained by solving Eqs. (12) and (13) for the volume fractions $\phi_{1}$ and $\phi_{2}$. These two nonlinear algebraic equations can be represented as

$f_{1}\left(a_{1}^{\mathrm{P}}, \phi_{1}, \phi_{2}, \phi_{3}, \chi_{12}, \chi_{13}, \chi_{23}\right)=0$
$f_{2}\left(a_{2}^{\mathrm{P}}, \phi_{1}, \phi_{2}, \phi_{3}, \chi_{12}, \chi_{13}, \chi_{23}\right)=0$

Clearly the volumes fractions must sum to unity:

$\phi_{1}+\phi_{2}+\phi_{3}=1$

The activities $a_{i}^{\mathrm{P}}$ are determined from Eqs. (5) and (14) as follows:

$a_{1}^{\mathrm{P}}=a_{1}^{\mathrm{L}}=x_{1} \gamma_{1}^{\mathrm{L}}=x_{1} \exp \left(\frac{A x_{2}^{2}}{R T}\right)$

$a_{2}^{\mathrm{P}}=a_{2}^{\mathrm{L}}=x_{2} \gamma_{2}^{\mathrm{L}}=x_{2} \exp \left(\frac{A x_{1}^{2}}{R T}\right)$

The interaction parameter $\chi_{12}$ is determined from Eq. (7), while the interaction parameters $\chi_{13}$ and $\chi_{23}$ are computed from Eqs. (9)-(11). The five nonlinear algebraic equations (10)-(13) and (17), are solved simultaneously to yield the volume fractions $\phi_{1}$ and $\phi_{2}$. We use the MATLAB nonlinear equation solver fsolve for this purpose.

To determine solubilities of the two components in the polymer, it is necessary to obtain the required physical property data and to estimate the unknown parameters of the solution model. The unknown parameters are the Margules constant $(A)$ and the six constants $\left(a_{1}, a_{2}, a_{3}, b_{1}, b_{2}, b_{3}\right)$ associated with the ternary interaction parameters. The Margules parameter is estimated from the nonlinear algebraic equation (6) using vapor-liquid equilibrium data for the binary liquid mixture. This equation can be represented as

$f_{3}\left(T, P, x_{1}, x_{2}, A\right)=0$

Given vapor-liquid equilibrium data over a range of liquid compositions, the constant $A$ is determined by solving the following nonlinear optimization problem:

$\min _{A} \sum_{j=1}^{N}\left[f_{3}\left(T_{j}, P_{j}, x_{1, j}, x_{2, j}, A\right)\right]^{2} \quad$ subject to

$0<A \leq A_{\mathrm{u}}$

where $N$ is the number of data points, $P_{j}, T_{j}, x_{1, j}$ and $x_{2, j}$ the experimental values of the pressure, temperature, component 1 mole fraction, and component 2 mole fraction, respectively, for the $j$ th data point, and $A_{\mathrm{u}}$ is an upper bound on the estimate of A. This problem is solved using the MATLAB nonlinear constrained optimization routine fmincon. 
Estimates of the six constants associated with the ternary interaction parameters are generated similarly. The nonlinear algebraic equations (10) and (11) can be represented as

$$
\begin{aligned}
& f_{4}\left(\chi_{13}, \chi_{13}^{\mathrm{b}}, \phi_{3}^{\mathrm{b} 1}, u_{2}, \phi_{3}, a_{1}, a_{2}, a_{3}\right)=0 \\
& f_{5}\left(\chi_{23}, \chi_{23}^{\mathrm{b}}, \phi_{3}^{\mathrm{b} 2}, u_{1}, \phi_{3}, b_{1}, b_{2}, b_{3}\right)=0
\end{aligned}
$$

Assume the availability of solubility data in mass of component $i$ sorbed per unit mass of polymer for the ternary mixture over a range of liquid compositions. Given the densities of the liquid components and the polymer, the binary solubility of component $i$ in mass of component $i$ sorbed per unit volume of polymer $\left(S_{i}^{\mathrm{b}}\right)$ and the ternary solubility of component $i$ in volume of component $i$ sorbed per unit volume of polymer $\left(S_{i}\right)$ are readily computed. The binary polymer volume fractions $\phi_{3}^{\mathrm{bi}}$ and binary interaction parameters $\chi_{i 3}^{\mathrm{b}}$ are calculated from the binary solubilities. The ternary solubilities are used to compute the ternary volume fractions $\phi_{i}$. The set of algebraic equations used to formulate the nonlinear least-squares estimation problem is obtained by combining Eqs. (20) and (21) with Eqs. (15)-(17). The decision variables in the optimization are the unknown constants $a_{1}, a_{2}, a_{3}, b_{1}$, $b_{2}$, and $b_{3}$. The optimization problem is solved using the MATLAB routine fmincon.

\subsubsection{Styrenelethylbenzene pervaporation membrane}

In previous research by the first author [4,5], PHS was used to synthesize a styrene selective pervaporation membrane for styrene/ethylbenzene separations. A polyurethane membrane of thickness $50 \mu \mathrm{m}$ was prepared by crosslinking PHS with multifunctional polyisocyanate. The permeation properties of the polyurethane membrane were investigated via sorption and flux experiments. Fig. 1 shows the styrene and ethylbenzene uptakes in the membrane as a function of the feed styrene concentration. The styrene uptake increases with increasing feed styrene concentration, while the ethylbenzene uptake exhibits a maximum due to the effects of membrane swelling.

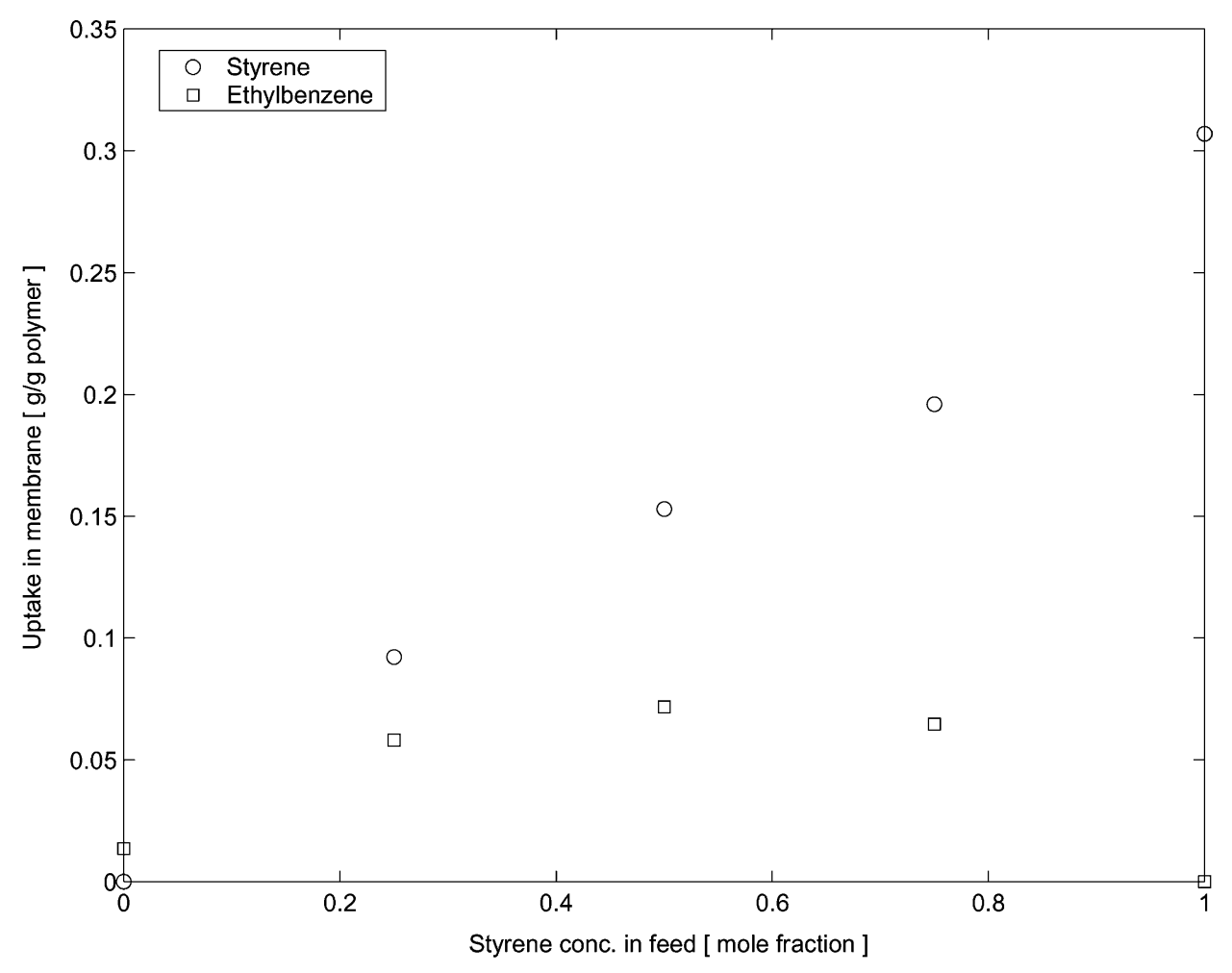

Fig. 1. Effect of feed concentration on styrene and ethylbenzene uptake in the polyurethane membrane. 
Table 1

Pure component physical property data

\begin{tabular}{lccc}
\hline Property & Styrene & Ethylbenzene & Polyurethane membrane \\
\hline Molar volume $\left(\mathrm{cm}^{3} / \mathrm{mol}\right)$ & 115.0 & 122.4 & - \\
Density $\left(\mathrm{g} / \mathrm{cm}^{3}\right)$ & 0.9060 & 0.8670 & 0.96 \\
Liquid viscosity $(\mathrm{cP})$ & 0.725 & 0.6428 & - \\
Heat capacity $(\mathrm{J} / \mathrm{g} \mathrm{K})$ & 1.6907 & 1.752 & - \\
Heat of vaporization $(\mathrm{J} / \mathrm{g})$ & 421.7 & 335.0 & - \\
Solubility $(\mathrm{g} / \mathrm{g}$ polymer) & 0.307 & 0.014 & - \\
\hline
\end{tabular}

Clearly the sorption mechanism favors styrene permeation.

The sorption data in Fig. 1 allow determination of the solution model parameters using the nonlinear estimation technique described above. Pure component physical property data are listed in Table 1 . The Margules constant $A$ is determined by solving the nonlinear optimization problem (19). The necessary vapor-liquid equilibrium data is shown in Table 2 along with the Antoine equations used to compute the pure component vapor pressures [6]. The estimate obtained is $A=163.9 \mathrm{~Pa} / \mathrm{mol} \mathrm{K}$.

The constants $a_{j}$ and $b_{j}$ are estimated from the data in Fig. 1 expressed as mass of component $i$ sorbed per unit mass of polymer. From these estimates the volume fractions $\phi_{i}$ of the ternary mixture are computed. Because there are only six data points available to estimate the six unknown parameters, there are no degrees of freedom for optimization. In this case, the parameter estimation problem is reduced to solving a set of coupled nonlinear algebraic equations. The resulting expressions for the ternary interaction parameters are as follows:

$$
\begin{aligned}
\chi_{13}= & 1.142-8.951 u_{2}^{2}+5.866 u_{2} \\
& -5.652\left(\phi_{3}-0.754\right)
\end{aligned}
$$

$$
\begin{aligned}
\chi_{23}= & 3.297+13.537 u_{1}^{2}-11.157 u_{1} \\
& +8.694\left(\phi_{3}-0.984\right)
\end{aligned}
$$

The predicted volume fractions obtained with the estimated parameters are shown in Fig. 2. The model provides very accurate predictions of this admittedly limited data set. Note that, the model captures the maximum in the ethylbenzene volume fraction. We have found that this effect cannot be captured with interaction parameter equations simpler than those in Eqs. (10) and (11).

\subsection{Diffusion model}

\subsubsection{Theory}

The component volume fractions obtained from the solution model are used to calculate component fluxes under the idealized conditions that the feed-side composition and temperature are constant and the permeate side of the membrane is maintained at vacuum. As shown in Section 2.3, these idealized fluxes can be used to compute membrane mass transfer coefficients that allow the calculation of component fluxes under the varying feed and permeate stream conditions encountered in a spiral wound pervaporation module.

\begin{tabular}{|c|c|c|c|c|c|c|c|c|}
\hline & \multicolumn{8}{|c|}{ Temperature $\left({ }^{\circ} \mathrm{C}\right)$} \\
\hline & 25.88 & 26.92 & 27.73 & 28.27 & 29.15 & 30.60 & 31.68 & 32.40 \\
\hline$x_{2}$ & 1.0 & 0.777 & 0.6505 & 0.575 & 0.433 & 0.222 & 0.0825 & 0.000 \\
\hline$y_{2}$ & 1.0 & 0.835 & 0.732 & 0.6625 & 0.535 & 0.310 & 0.128 & 0.000 \\
\hline
\end{tabular}
The diffusion model is based on the six parameter

Table 2

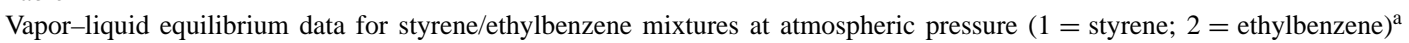




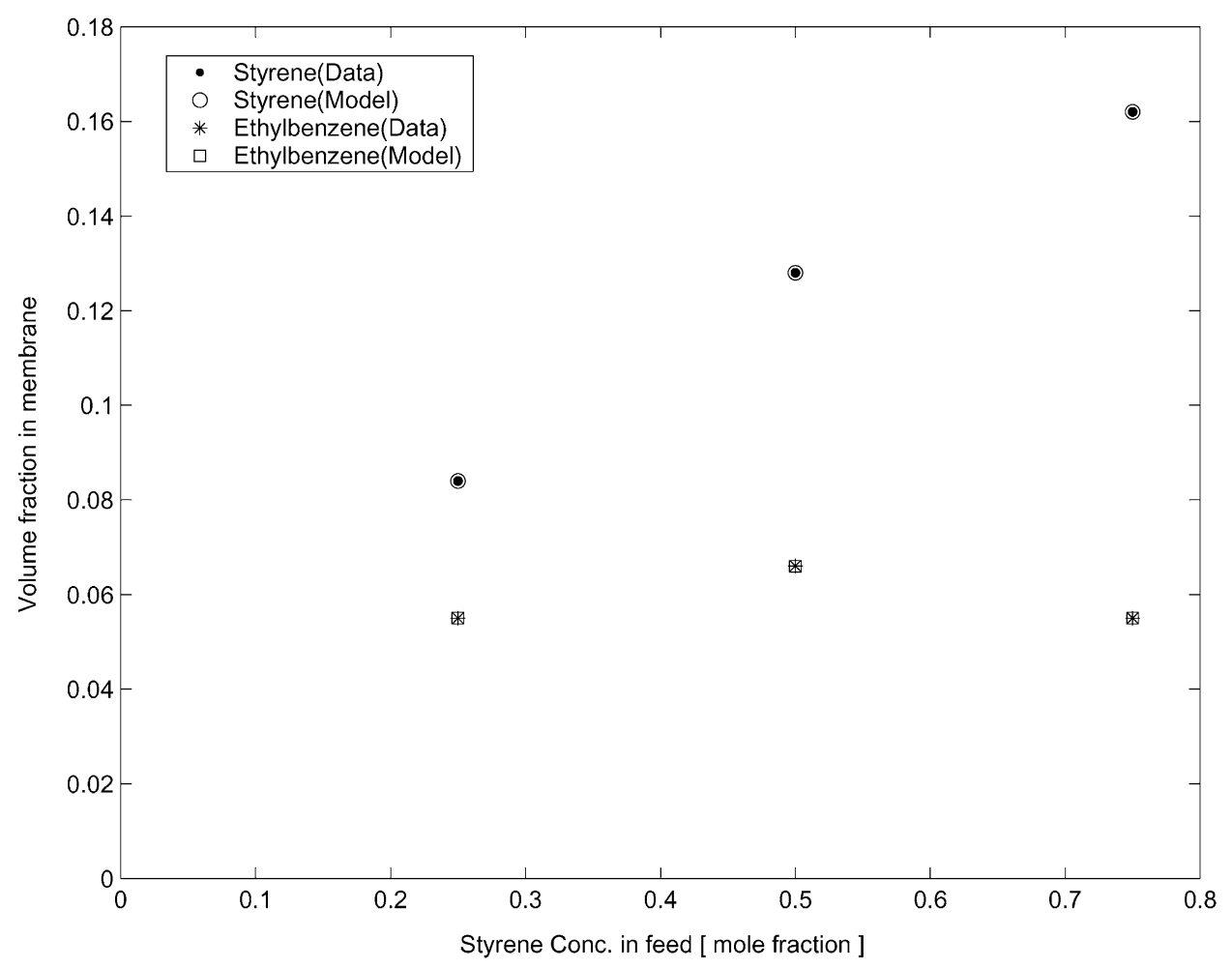

Fig. 2. Comparison of experimental and predicted solubilities of styrene and ethylbenzene in the polyurethane membrane.

diffusion coefficient equations for binary liquid mixtures proposed by Brun et al. [3]. Under the assumption of moderate membrane swelling, diffusion of a pure liquid in a polymer membrane can be described by the following form of Fick's law:

$J=-\frac{D}{1-\phi} \frac{\mathrm{d} C}{\mathrm{~d} z}$

where $J$ is the mass flux, $z$ the direction of the flux, $C$ the mass concentration of the diffusing species, $\phi$ the volume fraction of the species in the membrane, and $D$ is the diffusion coefficient. For binary liquid mixtures, Eq. (22) holds for each component $i$ :

$J_{i}=-\frac{D_{i}}{1-\phi_{i}} \frac{\mathrm{d} C_{i}}{\mathrm{~d} z}$

The diffusion coefficient $D_{i}$ may depend on the concentration of each component in the mixture. To account for this possibility, the identity $C_{i}=\rho_{i} \phi_{i}$ is used to rewrite Eq. (23) as
$J_{i} \mathrm{~d} z=-\frac{\rho_{i} D_{i}}{1-\phi_{i}} \mathrm{~d} \phi_{i}$

where $\rho_{i}$ is the density of component $i$. At $z=0$ the volume fraction $\phi_{i}$ is equal to the sorption value, which is denoted here as $\phi_{i}^{0}$. The volume fraction on the permeate side of the membrane is approximately zero if the permeate pressure is maintained near vacuum. Under the assumption that $D_{i}$ is constant, integration of Eq. (24) from $z=0$ to $z=l$ yields

$J_{i}^{*} l=\rho_{i} D_{i} \ln \left(1-\phi_{i}^{0}\right)$

where $l$ is the membrane thickness, and $J_{i}^{*}$ denotes the component flux obtained with a permeate vacuum. This equation suggests that a plot of $J_{i}^{*} l$ versus $\ln \left(1-\phi_{i}^{0}\right)$ should produce a straight line. If the relationship is significantly nonlinear, then $D_{i}$ is a function of the component concentrations in the membrane. In this case, the functional form of the concentration dependence can be deduced from the shape of the curve [7]. 




Fig. 3. Effect of feed concentration on flux and selectivity of the polyurethane membrane.

The procedure is illustrated for a polyurethane membrane of thickness $50 \mu \mathrm{m}$. Component fluxes $J_{i}^{*}$ are obtained from the flux and selectivity data [4] shown in Fig. 3. Note that the styrene selectivity decreases rapidly as the feed styrene concentration is increased due to a loss of sorption selectivity. This indicates that the polyurethane membrane is most appropriate for bulk separation of styrene and ethylbenzene. Fig. 4 suggests that the styrene diffusion coefficient has an exponential concentration dependence. An appropriate functional form for the concentration dependence of the ethylbenzene diffusion coefficient is less clear. Based on the styrene behavior, we utilize the six parameter diffusion coefficient model proposed by Brun et al. [3] for both components. The diffusion coefficients are assumed to depend exponentially on the concentration of each component:

$$
\begin{aligned}
& D_{1}=D_{1}^{0} \exp \left(A_{11} C_{1}+A_{12} C_{2}\right) \\
& D_{2}=D_{2}^{0} \exp \left(A_{21} C_{1}+A_{22} C_{2}\right)
\end{aligned}
$$

where $D_{i}^{0}$ are diffusion coefficients at infinite dilution, and the $A_{i j}$ are constant parameters. As shown below, these six parameters can be estimated from sorption and flux data.

\subsubsection{Estimation of the diffusion model parameters}

The equations used to compute the component fluxes are obtained by combining Eqs. (25) and (26) with Eq. (24):

$$
\begin{aligned}
& J_{1} \mathrm{~d} z=-D_{1}^{0} \rho_{1} \frac{\exp \left(A_{11} \phi_{1}+A_{12} \phi_{2}\right)}{1-\phi_{1}} \mathrm{~d} \phi_{1} \\
& J_{2} \mathrm{~d} z=-D_{2}^{0} \rho_{2} \frac{\exp \left(A_{21} \phi_{1}+A_{22} \phi_{2}\right)}{1-\phi_{2}} \mathrm{~d} \phi_{2}
\end{aligned}
$$

These equations are integrated from $z=0$ where $\phi_{i}=$ $\phi_{i}^{0}$ to $z=l$ where $\phi_{i}=0$ :

$$
J_{1}^{*}=-\frac{D_{1}^{0} \rho_{1}}{l} \int_{\phi_{1}^{0}}^{0} \frac{\exp \left(A_{11} \phi_{1}+A_{12} \phi_{2}\right)}{\left(1-\phi_{1}\right)} \mathrm{d} \phi_{1}
$$






Fig. 4. Concentration dependence of the styrene and ethylbenzene diffusion coefficients.

$J_{2}^{*}=-\frac{D_{2}^{0} \rho_{2}}{l} \int_{\phi_{2}^{0}}^{0} \frac{\exp \left(A_{21} \phi_{1}+A_{22} \phi_{2}\right)}{\left(1-\phi_{2}\right)} \mathrm{d} \phi_{2}$

Approximation of the integrals using Gaussian quadrature [10] yields

$$
\begin{aligned}
& J_{1}^{*} \approx-\frac{D_{1}^{0} \rho_{1}}{l} \sum_{k=1}^{M} \frac{\exp \left(A_{11} \phi_{1, k}+A_{12} \phi_{2, k}\right)}{\left(1-\phi_{1, k}\right)} w_{k} \\
& J_{2}^{*} \approx-\frac{D_{2}^{0} \rho_{2}}{l} \sum_{k=1}^{M} \frac{\exp \left(A_{21} \phi_{1, k}+A_{22} \phi_{2, k}\right)}{\left(1-\phi_{2, k}\right)} w_{k}
\end{aligned}
$$

where $M$ is the number of quadrature points, $w_{k}$ the quadrature weight at the quadrature point $\xi_{k}$, the quadrature points $\xi_{k} \in[0,1]$ are obtained as roots of the appropriate Jacobi polynomial, and

$$
\phi_{1, k}=\left(1-\xi_{k}\right) \phi_{1}^{0}, \quad \phi_{2, k}=\left(1-\xi_{k}\right) \phi_{2}^{0}
$$

Given the diffusion model parameters, the equations above allow the component fluxes $J_{i}^{*}$ to be computed from the volume fractions $\phi_{i}^{0}$ supplied by the solution model.

Parameters of the diffusion coefficient model are estimated from sorption and flux data. Given a set of $N$ data points $\left\{J_{1, j}^{*}, J_{2, j}^{*}, \phi_{1, j}^{0}, \phi_{2, j}^{0}\right\}$, the nonlinear algebraic equations (29) and (30) are rewritten for each data point to yield $2 N$ equations in the six unknown parameters $\theta=$ $\left[D_{1}^{0} D_{2}^{0} A_{11} A_{12} A_{21} A_{22}\right]^{\mathrm{T}}$. The parameters are determined by solving the nonlinear least-squares estimation problem:

$\min _{\theta} \sum_{j=1}^{N}\left[J_{j}^{*}-\hat{J}_{j}^{*}\right]^{\mathrm{T}}\left[J_{j}^{*}-\hat{J}_{j}^{*}\right]$

where $J_{j}^{*}=\left[J_{1, j}^{*} J_{2, j}^{*}\right]^{\mathrm{T}}$ and $\hat{J}_{j}^{*}=\left[\hat{J}_{1, j}^{*} \hat{J}_{2, j}^{*}\right]^{\mathrm{T}}$ are experimental and predicted values, respectively, of the component fluxes. The minimization is performed subject to nonlinear equality constraints derived from the $2 N$ component flux 
equations. We use the MATLAB routine fmincon to solve the constrained nonlinear optimization problem.

\subsubsection{Styrene/ethylbenzene pervaporation membrane}

The diffusion model is used to predict the styrene and ethylbenzene fluxes of a polyurethane membrane of $50 \mu \mathrm{m}$ thickness. The diffusion model parameters are estimated from the sorption and flux data in Figs. 1 and 3 , respectively. There are a total of 10 data points (styrene and ethylbenzene data for five styrene feed concentrations) available to estimate the six unknown parameters. The estimated parameter values are listed in Table 3. Styrene has a slightly higher diffusion coefficient at infinite dilution than does ethylbenzene. On the other hand, the ethylbenzene diffusion coefficient increases more rapidly with increasing ethylbenzene concentration than does the styrene diffusion coefficient with increasing styrene concentration. The ethylbenzene diffusion coefficient is significantly reduced by increasing styrene concentration, while the
Table 3

Diffusion coefficient model parameters

\begin{tabular}{llll}
\hline Component & $D^{0}\left(\mathrm{~m}^{2} / \mathrm{s}\right)$ & $A_{i i}$ & $A_{i j}$ \\
\hline Styrene & $8.78 \times 10^{-12}$ & 19.9 & -0.06 \\
Ethylbenzene & $8.13 \times 10^{-12}$ & 58.5 & -13.7 \\
\hline
\end{tabular}

styrene diffusion coefficient is virtually unaffected by the ethylbenzene concentration. As a result of this behavior, sorption rather than diffusion is the primary mechanism that controls the styrene permselectivity of the polyurethane membrane [4].

In Fig. 5, component flux data are compared to calculated fluxes derived from the estimated parameters in Table 3. The diffusion model provides accurate predictions of both fluxes given the diffusion coefficient form used for ethylbenzene and the limited number of data points available. Note that the model is able to predict the maximum in the ethylbenzene flux. We have found that this effect cannot be captured if the coupling terms $\left(A_{12}, A_{21}\right)$ are zero.

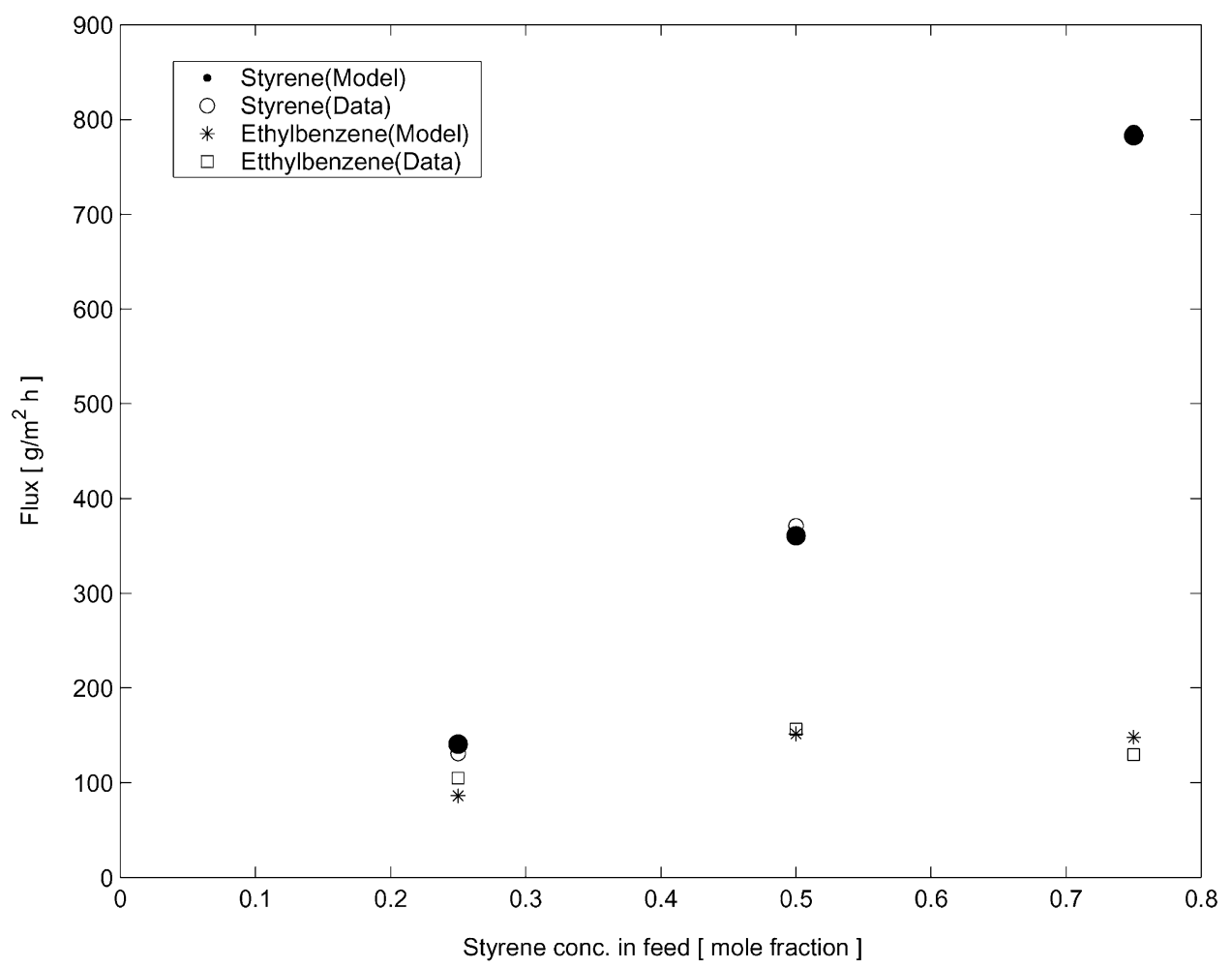

Fig. 5. Comparison of experimental and predicted component fluxes in the polyurethane membrane. 


\subsection{Overall mass transfer coefficient}

In addition to the polymer solution-diffusion properties, the permselectivity of a pervaporation membrane can be affected strongly by boundary layer resistances. It is generally accepted that the mass transfer resistance on the permeate side is negligible because the permeate stream is in the vapor phase. By contrast, the feed-side liquid boundary layer resistance can dominate the membrane resistance for low Reynolds number flows [19]. Using a simple resistance-in-series model [13], the overall mass transfer coefficients are calculated as

$\frac{1}{k_{\mathrm{t} i}}=\frac{1}{k_{\mathrm{m} i}}+\frac{1}{k_{1}}$

where $k_{\mathrm{t} i}$ is the overall mass transfer coefficient of component $i, k_{\mathrm{m} i}$ the membrane mass transfer coefficient of component $i$, and $k_{1}$ is the mass transfer coefficient associated with the liquid boundary layer.

The membrane mass transfer coefficient is computed as follows. Under the assumptions of negligible boundary layer resistance and ideal liquid and vapor behavior, $k_{\mathrm{m} i}$ is related to the component flux $J_{i}$ as

$J_{i}=k_{\mathrm{m} i}\left(x_{i} P_{i}^{\mathrm{sat}}-y_{i} P\right)$

where $k_{\mathrm{m} i}$ is expressed in units of $\mathrm{s} / \mathrm{m}, x_{i}$ and $y_{i}$ the liquid and vapor compositions, respectively, of component $i, P$ the permeate pressure and $P_{i}^{\text {sat }}$ is the saturation pressure of component $i$ at temperature $T$. Recall that the component flux $J_{i}^{*}$ in the solution-diffusion model is computed assuming the permeate side of the membrane is maintained at vacuum. This leads to the following simplification of Eq. (32):

$J_{i}^{*}=k_{\mathrm{m} i} x_{i} P_{i}^{\mathrm{sat}}$

The idealized component flux $J_{i}^{*}$ is computed at a fixed reference temperature $T_{0}$. The following phenomenological relation [20] is used to account for temperature variations encountered in a spiral wound pervaporation module:

$J_{i}^{*}(T)=J_{i}^{*}\left(T_{0}\right) \exp \left[-\frac{E_{i}}{R}\left(\frac{1}{T}-\frac{1}{T_{0}}\right)\right]$

where $J_{i}^{*}\left(T_{0}\right)$ is the idealized flux of component $i$ that is obtained from the solution-diffusion model, $J_{i}^{*}(T)$ the temperature corrected idealized flux of component $i$, and $E_{i}$ is the activation energy of component $i$. This relation suggests that a plot of $\ln J_{i}^{*}(T)$ versus $(1 / T)-\left(1 / T_{0}\right)$ should yield a straight line with slope $-\left(E_{i} / R\right)$. Fig. 6 illustrates the procedure for a polyurethane membrane of thickness $50 \mu \mathrm{m}$. The required temperature-dependent flux data are obtained from [4] for a feed styrene mole fraction $x_{\mathrm{f}}=0.5$. The reference temperature is chosen as $T_{0}=25^{\circ} \mathrm{C}$. Both styrene and ethylbenzene exhibit an exponential dependence on temperature. The estimated activation energies listed in Table 4 demonstrate that the ethylbenzene flux is more strongly affected by temperature than is the styrene flux. The membrane mass transfer coefficient is calculated from the temperature corrected idealized flux as follows:

$k_{\mathrm{m} i}=\frac{J_{i}^{*}(T)}{x_{i} P_{i}^{\mathrm{sat}}}$

It is important to emphasize that Eq. (35) is used only for calculation of $k_{\mathrm{m} i}$.

The liquid boundary layer resistance is estimated from the following empirical relations [1]:

- local value for laminar flow:

$$
S h=\frac{\tilde{k}_{1} x}{D_{12}^{1}}=0.33 R e^{1 / 2} S c^{1 / 3}
$$

- average value for laminar flow:

$$
S h_{\mathrm{L}}=\frac{\hat{k}_{1} L}{D_{12}^{1}}=0.66 R e_{\mathrm{L}}^{1 / 2} S c^{1 / 3}
$$

- local value for turbulent flow:

$$
S h=\frac{\tilde{k}_{1} x}{D_{12}^{1}}=0.0292 R e^{4 / 5} S c^{1 / 3}
$$

- average value for turbulent flow:

$$
S h_{\mathrm{L}}=\frac{\hat{k}_{1} L}{D_{12}^{1}}=0.0365 \operatorname{Re}_{\mathrm{L}}^{4 / 5} S c^{1 / 3}
$$

where $S h$ and $S h_{\mathrm{L}}$ are the local and average Sherwood numbers, respectively, $\tilde{k}_{1}$ and $\hat{k}_{1}$ the local and average liquid boundary layer mass transfer coefficients, respectively, expressed in units of $\mathrm{m} / \mathrm{s}, x$ the distance from the module entrance, $L$ the length of the module, and $D_{12}^{1}$ is the diffusion coefficient of the two liquid phase components calculated using the method 


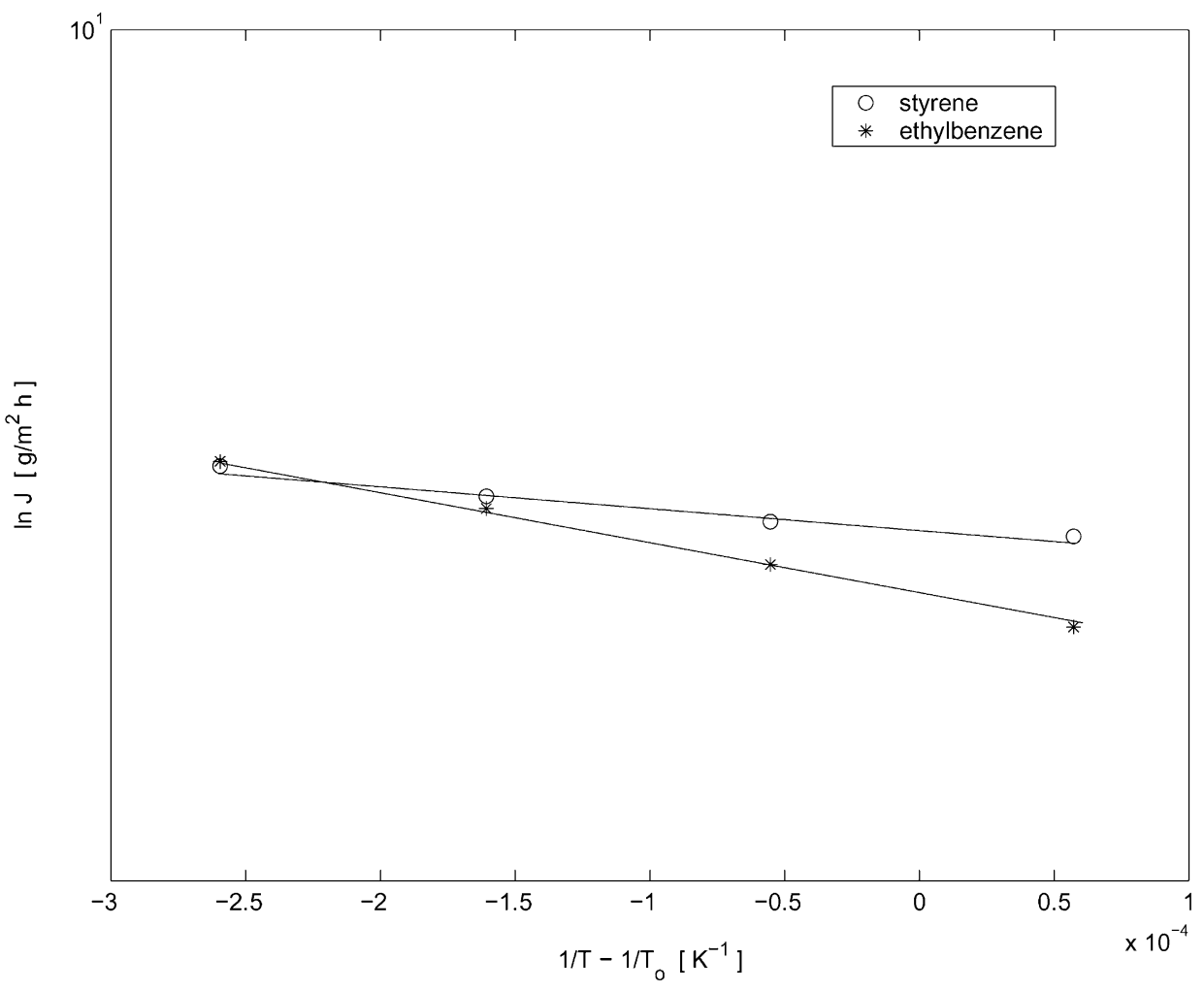

Fig. 6. Temperature dependence of the styrene and ethylbenzene fluxes.

Table 4

Model parameters for temperature-dependent flux

\begin{tabular}{llc}
\hline Component & $E(\mathrm{~J} / \mathrm{mol})$ & $J_{0}\left(\mathrm{~kg} / \mathrm{m}^{2} \mathrm{~h}\right)$ \\
\hline Styrene & $1.343 \times 10^{4}$ & 17.286 \\
Ethylbenzene & $2.986 \times 10^{4}$ & 7.147 \\
\hline
\end{tabular}

in [23]. The Reynolds numbers $R e$ and $R e_{\mathrm{L}}$ and the Schmidt number $S c$ are defined as

$R e=\frac{x v \rho_{1}}{\mu_{1}}, \quad R e_{\mathrm{L}}=\frac{L v \rho_{1}}{\mu_{1}}, \quad S c=\frac{\mu_{1}}{\rho_{1} D_{12}^{\mathrm{L}}}$

where $v$ is the feed-side liquid stream velocity, and $\mu_{1}$ and $\rho_{1}$ are the viscosity and density, respectively, of the liquid. Because the feed-side properties vary in the $x$-direction, the local mass transfer coefficient $\tilde{k}_{1}$ is used to calculate the overall mass transfer coefficient $k_{\mathrm{t} i}$. The average mass transfer coefficient $\hat{k}_{1}$ is introduced simply for convenience in presenting the subsequent simulation results. Note that the local and average mass transfer coefficients are different even at $x=L$.

The overall mass transfer coefficient is used to calculate component fluxes for operating conditions encountered in an actual spiral wound pervaporation module with non-zero permeate pressure and liquid boundary layer resistance. The component flux equation is a straightforward generalization of Eq. (32):

$J_{i}=k_{\mathrm{t} i}\left(x_{i} P_{i}^{\mathrm{sat}}-y_{i} P\right)$

where $k_{\mathrm{t} i}$ is expressed in units of $\mathrm{s} / \mathrm{m}$. It is important to emphasize that the component fluxes vary with both the feed-side and permeate-side stream conditions.

\section{Spiral wound pervaporation model}

To predict the separation performance of a spiral wound pervaporation module, the solution-diffusion 
model must be combined with mass, momentum and energy balances that govern the module transport behavior. The component fluxes produced by the solution-diffusion model vary with the feed-side and permeate-side stream conditions. Therefore, transport equations must be derived for both the feed and permeate sides of the module. For bulk hydrocarbon separations, the proposed model will yield more accurate predictions than the simpler pervaporation model in [13], that is based on a constant mass transfer coefficient and includes only permeate-side balances. The feed-side and permeate-side transport equations are derived in Section 3.1. The resulting model consists of a coupled set of nonlinear ordinary differential equations with two spatial coordinates and mixed boundary conditions. A numerical procedure for solving the pervaporation model is presented in Section 3.2.

\subsection{Module transport equations}

Fig. 7 depicts permeation of a binary mixture through an extended membrane leaf of a spiral wound pervaporation module. The bulk feed flow is in the $x$-direction while the bulk permeate flow is the $y$-direction. Permeation through the membrane takes place in the $z$-direction. The mass transfer driving force is reduced along the $x$-direction as the feed becomes depleted in the more permeable component, while it is increased along the $y$-direction as the permeate pressure decreases. Because there is momentum transport in the $z$-direction due to flux through the membrane, the feed-side and permeate-side stream properties can be viewed as varying with respect to all three spatial coordinates. This would yield a very complex modeling problem and the resulting partial differential equation model would not be amenable to numerical solution.

In this paper, the module transport equations are derived under the following simplifying assumptions:

1. Feed-side variations in the $y$-direction are small.

2. Permeate-side variations in the $x$-direction are small.

3. Feed-side and permeate-side variables can be averaged with respect to the $z$-direction.

4. Feed-side and permeate-side diffusion are negligible compared to convection.

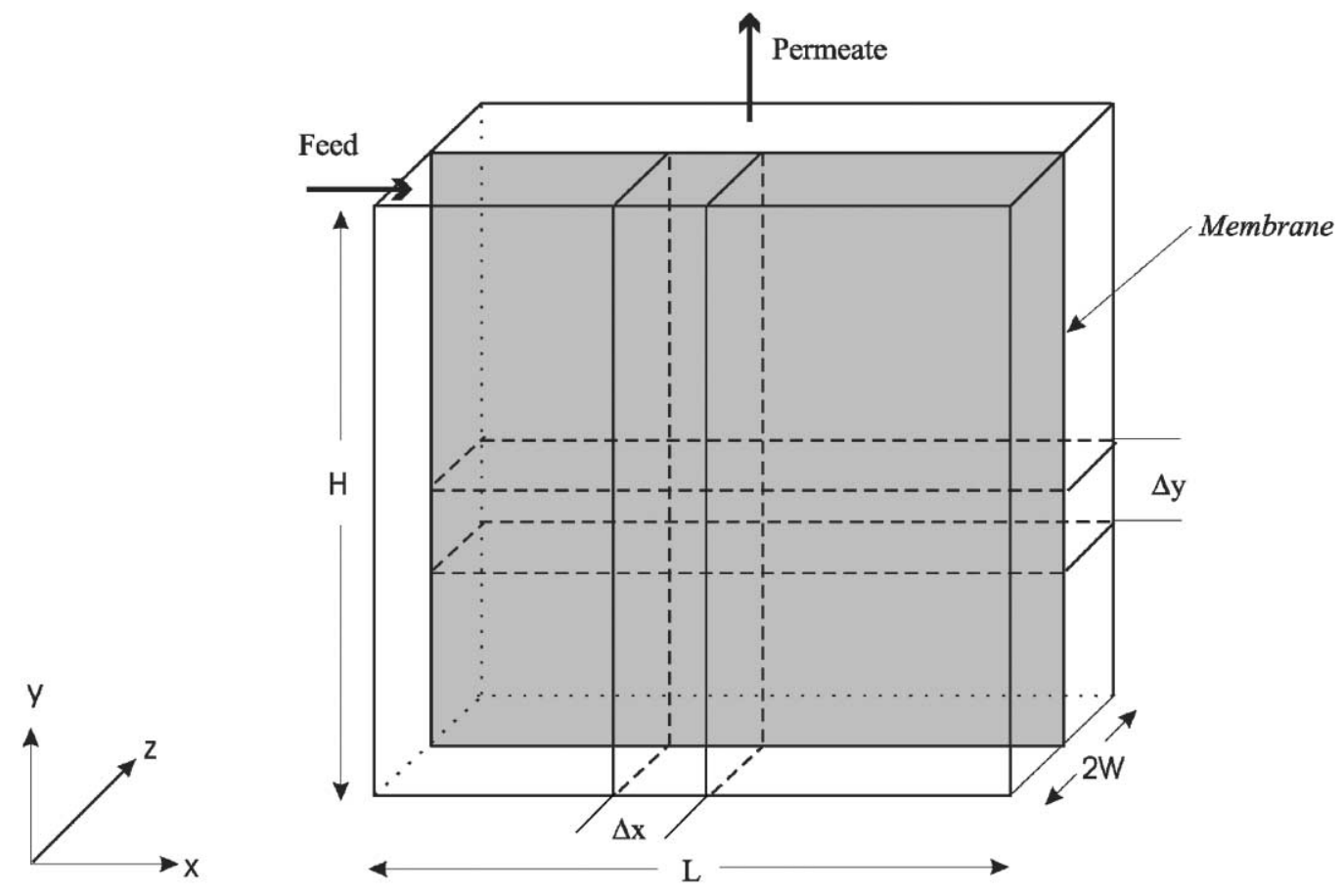

Fig. 7. Extended membrane leaf of a spiral wound pervaporation module. 
5. The feed liquid is an ideal solution.

6. The permeate vapor is an ideal gas.

The first two assumptions imply that feed-side and permeate-side variables vary primarily in the direction of bulk feed and permeate flow, respectively. This is a reasonable simplification for momentum related variables such as velocity. These assumptions may appear to be questionable for the feed and permeate concentrations since the driving force for mass transfer varies with respect to both flow directions. This effect is captured in the transport model by allowing the component fluxes to vary with respect to both $x$ and $y$. Therefore, the feed-side concentration and permeate-side concentration depend indirectly on the $y$-direction and $x$-direction, respectively, due to their coupling through the component fluxes. It is worth noting that these assumptions also are invoked by Hickey and Gooding [13] in their spiral wound pervaporation model for removal of volatile organic compounds from water. The third assumption is commonly used in transport models where the description of such spatial variations is considered unnecessary. The fourth assumption is reasonable for convection dominated flows that are expected in bulk hydrocarbon separations. The validity of the fifth assumption is dependent on the particular components in the binary mixture. It is a reasonable simplification for the styrene/ethylbenzene mixtures considered in this paper [6]. The sixth assumption is reasonable due to the low pressures and moderate temperatures typically present on the permeate side of the module.

\subsubsection{Feed-side balances}

First, the feed-side momentum balance is derived. To generate a suitable differential equation for the feed-side velocity, it is necessary to determine the feed-side pressure drop that results from flux across the membrane. Because permeation occurs in the $z$-direction, the $x$-component of the velocity $v_{x}$ varies with $z$ and the $z$-component of the velocity $v_{z}$ is non-zero. However, $v_{z} \ll v_{x}$, since, the permeation flux typically is much smaller than the convective flux. Also note that feed-side flow takes place in a narrow channel, since the module length $L$ is much greater than the width $W$. These conditions allow the use of the lubrication approximation under which the feed-side momentum balance is reduced to [8] $\frac{\partial^{2} v_{x}}{\partial z^{2}}=\frac{1}{\mu_{1}} \frac{\mathrm{d} P}{\mathrm{~d} x}$

where the feed-side pressure $P$ is independent of $z$ and the liquid viscosity $\mu_{1}$ is assumed to be constant. When combined with a feed-side mass balance and appropriate boundary conditions on the velocity, this equation allows the calculation of both $v_{x}(x, z)$ and $P(x)$. As shown in Appendix A, the following equations for the pressure and the $z$-averaged velocity $v(x)$ are obtained by neglecting frictional effects:

$\frac{\mathrm{d} P}{\mathrm{~d} x}=-\frac{12 \mu_{1} v}{W^{2}}$

$\frac{\mathrm{d} v}{\mathrm{~d} x}=-\frac{2 \tilde{J} M_{\mathrm{p}}}{\rho_{\mathrm{p}} W}$

where $\tilde{J}$ is the total molar flux through the membrane, and $W$ is the width of the membrane element. The molecular weight $M_{\mathrm{p}}$ and density $\rho_{\mathrm{p}}$ of the local permeate are computed from the component molar fluxes $\tilde{J}_{1}$ and $\tilde{J}_{2}$ :

$M_{\mathrm{p}}=\frac{M_{1} \tilde{J}_{1}+M_{2} \tilde{J}_{2}}{\tilde{J}_{1}+\tilde{J}_{2}}$

$\rho_{\mathrm{p}}=\frac{M_{1} \tilde{J}_{1}+M_{2} \tilde{J}_{2}}{\left(M_{1} \tilde{J}_{1} / \rho_{1}\right)+\left(M_{2} \tilde{J}_{2} / \rho_{2}\right)}$

where $M_{1}$ and $M_{2}$ are the component molecular weights, and $\rho_{1}$ and $\rho_{2}$ are the pure component densities. The molar fluxes are related to the mass fluxes introduced in the solution-diffusion model as

$$
\begin{aligned}
& \tilde{J}_{1}=\frac{J_{1}}{M_{1}}, \quad \tilde{J}_{2}=\frac{J_{2}}{M_{2}}, \\
& \tilde{J}=\tilde{J}_{1}+\tilde{J}_{2}=\frac{J_{1}}{M_{1}}+\frac{J_{2}}{M_{2}}
\end{aligned}
$$

A feed-side mass balance on component $i$ over the differential element $\Delta x$ and $\Delta y$ yields

$W \Delta y\left(\left.n_{i x}\right|_{x}-\left.n_{i x}\right|_{x+\Delta x}\right)-2 \Delta x \Delta y \tilde{J}_{i}=0$

where $n_{i x}$ is the feed-side component molar flux. Dividing by $\Delta x \Delta y$ and taking the limit as $\Delta x \rightarrow 0$ yields

$-W \frac{\partial n_{i x}}{\partial x}-2 \tilde{J}_{i}=0$

The convective flux can be expressed as

$n_{i x}=x_{i} n_{x}=x_{i} \tilde{\rho}_{1} v$ 
where $n_{x}$ is the total feed-side molar flux, and $x_{i}$ is the feed-side component mole fraction. The liquid molar density $\tilde{\rho}_{1}$ can be expressed as

$$
\begin{aligned}
\tilde{\rho}_{1} & =\frac{\rho_{1}}{M_{1}} \\
& =\frac{\left(M_{1} x_{1}+M_{2} x_{2}\right) /\left(\left(M_{1} x_{1} / \rho_{1}\right)+\left(M_{2} x_{2} / \rho_{2}\right)\right)}{M_{1} x_{1}+M_{2} x_{2}} \\
& =\frac{1}{\left(M_{1} x_{1} / \rho_{1}\right)+\left(M_{2} x_{2} / \rho_{2}\right)}
\end{aligned}
$$

where $\rho_{1}$ is the liquid mass density, and $M_{1}$ is the liquid molecular weight. Substitution of Eqs. (44) and (45) into Eq. (43) yields

$$
\begin{aligned}
& \frac{x_{i}}{\left(M_{i} x_{i} / \rho_{i}\right)+\left(M_{j}\left(1-x_{i}\right) / \rho_{j}\right)} \frac{\mathrm{d} v}{\mathrm{~d} x} \\
& +\frac{M_{j}}{\rho_{j}} \frac{v}{\left[\left(M_{i} x_{i} / \rho_{i}\right)+\left(M_{j}\left(1-x_{i}\right) / \rho_{j}\right)\right]^{2}} \frac{\mathrm{d} x_{i}}{\mathrm{~d} x} \\
& =-\frac{2 \tilde{J}_{i}}{W}
\end{aligned}
$$

The feed-side differential energy balance is written as

$$
\begin{aligned}
& W \Delta y\left[\left.\rho_{1} C_{p}^{\mathrm{L}} v\left(T-T_{0}\right)\right|_{x}-\left.\rho_{1} C_{p}^{\mathrm{L}} v\left(T-T_{0}\right)\right|_{x+\Delta x}\right] \\
& \quad+2 \Delta x \Delta y J\left[\lambda+C_{p}^{\mathrm{L}}\left(T-T_{0}\right)\right]=0
\end{aligned}
$$

where $T$ is the feed-side temperature, $C_{p}^{\mathrm{L}}$ and $\lambda$ the heat capacity and latent heat of vaporization, respectively, of the liquid mixture, $T_{0}$ the reference temperature, and the mass flux $J$ is related to the molar flux $\tilde{J}$ as in Eq. (42). This equation is derived by equating the energy removed from the feed stream to the energy required to vaporize the permeate which fluxes through the membrane. The heat capacity and heat of vaporization are assumed to be constant for simplicity. Dividing Eq. (47) by $\Delta x \Delta y$ and taking the limit as $\Delta x \rightarrow 0$ yields

$$
\frac{\mathrm{d}}{\mathrm{d} x}\left[\rho_{1} v\left(T-T_{0}\right)\right]=\frac{2 J\left[\lambda+C_{p}^{\mathrm{L}}\left(T-T_{0}\right)\right]}{W C_{p}^{\mathrm{L}}}
$$

Expansion of the derivative yields

$$
\begin{gathered}
v\left(T-T_{0}\right) \frac{\mathrm{d} \rho_{\mathrm{l}}}{\mathrm{d} x_{i}} \frac{\mathrm{d} x_{i}}{\mathrm{~d} x}+\rho_{\mathrm{l}}\left(T-T_{0}\right) \frac{\mathrm{d} v}{\mathrm{~d} x}+\rho_{\mathrm{l}} v \frac{\mathrm{d} T}{\mathrm{~d} x} \\
=\frac{2 J\left[\lambda+C_{p}^{\mathrm{L}}\left(T-T_{0}\right)\right]}{W C_{p}^{\mathrm{L}}}
\end{gathered}
$$

where $\mathrm{d} \rho_{1} / \mathrm{d} x_{i}$ can be evaluated from Eq. (45). The differential equations (39), (46) and (48) for the feed-side velocity, composition and temperature are subject to the following boundary conditions:

$$
v(0)=v_{\mathrm{f}}, \quad x_{i}(0)=x_{\mathrm{f}}, \quad T(0)=T_{\mathrm{f}}
$$

where $x=0$ denotes the location where feed is introduced to the module, and $v_{\mathrm{f}}, x_{\mathrm{f}}$ and $T_{\mathrm{f}}$ are the velocity, composition and temperature, respectively, of the feed stream.

\subsubsection{Permeate-side balances}

A permeate-side mass balance on component $i$ over the differential element $\Delta x$ and $\Delta y$ is written as

$W \Delta x\left(\left.m_{i y}\right|_{y}-\left.m_{i y}\right|_{y+\Delta y}\right)+2 \Delta x \Delta y \tilde{J}_{i}=0$

where $m_{i y}$ is the permeate-side component molar flux. Dividing by $\Delta x \Delta y$ and taking the limit as $\Delta y \rightarrow 0$ yields

$-W \frac{\mathrm{d} m_{i y}}{\mathrm{~d} y}+2 \tilde{J}_{i}=0$

The convective flux can be expressed as

$m_{i y}=y_{i} m_{y}=y_{i} \tilde{\rho}_{\mathrm{v}} u$

where $m_{y}$ is the total permeate-side molar flux, $y_{i}$ the permeate-side component mole fraction, and $u$ is the permeate-side velocity. The vapor molar density $\tilde{\rho}_{\mathrm{v}}$ is calculated assuming ideal gas behavior:

$\tilde{\rho}_{\mathrm{v}}=\frac{P}{R T_{\mathrm{p}}}$

where $P$ and $T_{\mathrm{p}}$ are the permeate-side pressure and temperature, respectively. Substitution of Eqs. (50) and (51) into Eq. (49) yields

$P u \frac{\mathrm{d} y_{i}}{\mathrm{~d} y}+y_{i} P \frac{\mathrm{d} u}{\mathrm{~d} y}+y_{i} u \frac{\mathrm{d} P}{\mathrm{~d} y}=\frac{2 \tilde{J}_{i} R T}{W}$

A permeate-side differential mass balance is written as $W \Delta x\left(\left.\rho_{\mathrm{v}} u\right|_{y}-\left.\rho_{\mathrm{v}} u\right|_{y+\Delta y}\right)+2 \Delta x \Delta y \tilde{J} M_{\mathrm{v}}=0$

where the vapor molecular weight $M_{\mathrm{v}}$ and vapor mass density $\rho_{\mathrm{v}}$ are calculated as

$$
\begin{aligned}
& M_{\mathrm{v}}=M_{1} y_{1}+M_{2} y_{2} \\
& \rho_{\mathrm{v}}=\tilde{\rho}_{\mathrm{v}} M_{\mathrm{v}}=\frac{P}{R T_{\mathrm{p}}} M_{\mathrm{v}}
\end{aligned}
$$


Dividing by $\Delta x \Delta y$ and taking the limit as $\Delta y \rightarrow 0$ yields

$$
-\frac{\mathrm{d}\left(\rho_{\mathrm{v}} u\right)}{\mathrm{d} y}+\frac{2 M_{\mathrm{v}} \tilde{J}}{W}=0
$$

Substitution of Eqs. (53) and (54) into Eq. (55) and expansion of the derivative produces

$$
\begin{gathered}
P u\left(M_{i}-M_{j}\right) \frac{\mathrm{d} y_{i}}{\mathrm{~d} y}+P M_{\mathrm{v}} \frac{\mathrm{d} u}{\mathrm{~d} y}+u M_{\mathrm{v}} \frac{\mathrm{d} P}{\mathrm{~d} y} \\
=\frac{2 M_{\mathrm{v}} \tilde{J} R T}{W}
\end{gathered}
$$

The permeate-side momentum balance can be written as [2]

$$
\frac{\mathrm{d}\left(\rho_{\mathrm{v}} u^{2}\right)}{\mathrm{d} y}+\frac{\mathrm{d} \tau_{y y}}{\mathrm{~d} y}+\frac{\mathrm{d} P}{\mathrm{~d} y}=0
$$

where the tensor $\tau_{y y}$ depends on the Fanning friction factor $f$ as

$\frac{\mathrm{d} \tau_{y y}}{\mathrm{~d} y}=\frac{2 \rho_{\mathrm{v}} u^{2}}{W} f$

The friction factor is computed as [2]

$f=\frac{A}{R e^{n}}, \quad R e=\frac{W \rho_{\mathrm{v}} u}{\mu_{\mathrm{v}}}$

where $A$ is a constant that depends on the module geometry, and $\mu_{\mathrm{v}}$ is the viscosity of the vapor. In the subsequent simulations, the parameters are chosen as $n=$ 1 and $A=24$. Substitution of Eqs. (53), (54) and (58) into Eq. (57) and expansion of the derivatives produces

$$
\begin{aligned}
& \left(M_{i}-M_{j}\right) P u^{2} \frac{\mathrm{d} y_{i}}{\mathrm{~d} y}+2 u P M_{\mathrm{v}} \frac{\mathrm{d} u}{\mathrm{~d} y} \\
& +\left(M_{\mathrm{v}} u^{2}+R T_{\mathrm{p}}\right) \frac{\mathrm{d} P}{\mathrm{~d} y}+\frac{2 P M_{\mathrm{v}} u^{2}}{W} f=0
\end{aligned}
$$

The differential equations (52) (56) and (59) for the permeate-side composition, velocity and pressure are subject to the following boundary conditions:

$y_{i}(0)=\frac{\tilde{J}_{i}(0)}{\tilde{J}(0)}, \quad u(0)=0, \quad P(H)=P_{H}$

where $y=0$ and $y=H$ denote the location of the closed end and collection tube, respectively, on the permeate side of the module, and $P_{H}$ denotes the permeate pressure in the collection tube. The boundary condition for the composition is determined by the ratio of local molar fluxes because there is no bulk permeate stream at the closed end of the module.

\subsection{Model solution}

The pervaporation model consists of the six nonlinear ordinary differential equations (39), (46), (48), (52), (56) and (59) with two spatial coordinates and mixed boundary conditions. In this section, we present a numerical solution procedure that yields predictions of the feed-side velocity, composition and temperature and the permeate-side composition, velocity and pressure as a function of the feed and permeate flow directions. First, the model is simplified such that each differential equation contains a single derivative. Straightforward but laborious algebraic manipulations yield the following model equations:

$\frac{\mathrm{d} x_{i}}{\mathrm{~d} x}=\frac{\rho_{i} V_{1}\left(2 \tilde{J} M_{\mathrm{p}} x_{i}-2 \tilde{J}_{i} V_{1} \rho_{\mathrm{p}}\right)}{M_{j} v W \rho_{\mathrm{p}}}$

$\frac{\mathrm{d} v}{\mathrm{~d} x}=-\frac{2 \tilde{J} M_{\mathrm{p}}}{W \rho_{\mathrm{p}}}$

$\frac{\mathrm{d} T}{\mathrm{~d} x}=-\frac{2 J M_{\mathrm{p}} \lambda}{\rho_{\mathrm{l}} v W C_{p}^{\mathrm{l}}}$

$\frac{\mathrm{d} y_{i}}{\mathrm{~d} y}=\frac{2 \tilde{J}_{i} R T_{\mathrm{p}} M_{\mathrm{v}}-2 \tilde{J} R T_{\mathrm{p}} M_{\mathrm{p}} y_{i}}{W P u M_{j}}$

$$
\begin{gathered}
\frac{\mathrm{d} u}{\mathrm{~d} y}=\frac{2 R T_{\mathrm{p}}\left(R T_{\mathrm{p}} \tilde{J}_{i} M_{\mathrm{v}}-R T_{\mathrm{p}} \tilde{J}_{i} M_{j}-R T_{\mathrm{p}} \tilde{J} y_{i} M_{\mathrm{p}}-\tilde{J} u^{2} M_{\mathrm{p}} M_{j} y_{i}\right)-2 P M_{\mathrm{v}} u^{3} f y_{i} M_{j}}{W P y_{i} M_{j}\left(M_{\mathrm{v}} u^{2}-R T_{\mathrm{p}}\right)} \\
\frac{\mathrm{d} P}{\mathrm{~d} y}=\frac{2 R T_{\mathrm{p}} u\left(M_{\mathrm{v}} \tilde{J} M_{\mathrm{p}} y_{i}-M_{\mathrm{v}}^{2} \tilde{J}_{i}+M_{j} \tilde{J} M_{\mathrm{p}} y_{i}+M_{j} \tilde{J}_{i} M_{\mathrm{v}}\right)+2 P M_{\mathrm{v}} u^{2} f y_{i} M_{j}}{W y_{i} M_{j}\left(M_{\mathrm{v}} u^{2}-R T_{\mathrm{p}}\right)}
\end{gathered}
$$


where the specific volume of the liquid $V_{1}$ is defined as

$V_{1}=\frac{1}{\rho_{1}}=\frac{M_{i} x_{i}}{\rho_{i}}+\frac{M_{2} x_{2}}{\rho_{2}}$

Next the model equations are non-dimensionalized to improve scaling and to reduce the number of parameters. Utilizing the scaling factors introduced in [13], the dimensionless variables are defined as

$x^{*}=\frac{x}{L}, \quad y^{*}=\frac{y}{H}, \quad x_{i}^{*}=x_{i}$,

$v^{*}=\frac{v}{v_{\mathrm{f}}}, \quad T^{*}=\frac{T}{T_{\mathrm{f}}}$,

$y_{i}^{*}=y_{i}, \quad u^{*}=u \sqrt{\frac{M_{1}}{R T_{\mathrm{p}}}}, \quad P^{*}=\frac{P}{P_{1}^{\mathrm{sat}}}$

where the subscript 1 denotes the first component, and $T_{\mathrm{p}}$ is the permeate temperature. The dimensionless model equations are as follows:

$\frac{\mathrm{d} x_{i}^{*}}{\mathrm{~d} x^{*}}=\frac{L \rho_{i} V_{1}\left(2 \tilde{J} M_{\mathrm{p}} x_{i}^{*}-2 \tilde{J}_{i} V_{1} \rho_{\mathrm{p}}\right)}{M_{j} v^{*} v_{\mathrm{f}} W \rho_{\mathrm{p}}}$

$\frac{\mathrm{d} v^{*}}{\mathrm{~d} x^{*}}=-\frac{2 L \tilde{J} M_{\mathrm{p}}}{v_{\mathrm{f}} W \rho_{\mathrm{p}}}$

$\frac{\mathrm{d} T^{*}}{\mathrm{~d} x^{*}}=-\frac{2 L J M_{\mathrm{p}} \lambda}{\rho_{\mathrm{l}} v^{*} v_{\mathrm{f}} W C_{p}^{\mathrm{l}} T_{\mathrm{f}}}$

$\frac{\mathrm{d} y_{i}^{*}}{\mathrm{~d} y^{*}}=\frac{H \sqrt{M_{1} / R T_{\mathrm{p}}}\left(2 \tilde{J}_{i} R T_{\mathrm{p}} M_{\mathrm{v}}-2 \tilde{J} R T_{\mathrm{p}} M_{\mathrm{p}} y_{i}^{*}\right)}{W P^{*} P_{1}^{\mathrm{sat}} u^{*} M_{j}}$

$\frac{\mathrm{d} u^{*}}{\mathrm{~d} y^{*}}=-H \sqrt{\frac{M_{1}}{R T_{\mathrm{p}}}} \frac{2 R T_{\mathrm{p}}\left(R T_{\mathrm{p}} \tilde{J}_{i} M_{\mathrm{v}}-R T_{\mathrm{p}} \tilde{J}_{i} M_{j}-R T_{\mathrm{p}} \tilde{J} y_{i}^{*} M_{\mathrm{p}}-\tilde{J} u^{2} M_{\mathrm{p}} M_{i} y_{i}^{*}\right)-2 P^{*} P_{1}^{\mathrm{sat}} M_{\mathrm{v}} u^{3} f y_{i}^{*} M_{j}}{W P^{*} P_{1}^{\mathrm{sat}} y_{i}^{*} M_{j}\left(M_{\mathrm{v}} u^{2}-R T_{\mathrm{p}}\right)}$

$\frac{\mathrm{d} P^{*}}{\mathrm{~d} y^{*}}=H \frac{2 R T_{\mathrm{p}} u\left(M_{\mathrm{v}} \tilde{J} M_{\mathrm{p}} y_{i}^{*}-M_{\mathrm{v}}^{2} \tilde{J}_{i}+M_{j} \tilde{J} M_{\mathrm{p}} y_{i}^{*}+M_{j} \tilde{J}_{i} M_{\mathrm{v}}\right)+2 P^{*} P_{1}^{\mathrm{sat}} M_{\mathrm{v}} u^{2} f y_{i}^{*} M_{j}}{W P_{1}^{\mathrm{sat}} y_{i}^{*} M_{j}\left(M_{\mathrm{v}} u^{2}-R T_{\mathrm{p}}\right)}$

where the mass component flux (36) expressed in terms of the dimensionless variables is

$J_{i}=k_{\mathrm{t} i}\left(x_{i}^{*} P_{i}^{\mathrm{sat}}-y_{i}^{*} P^{*} P_{1}^{\mathrm{sat}}\right)$

Recall that the molar component fluxes $\tilde{J}_{i}$ can be computed from $J_{i}$ as in Eq. (42). The boundary conditions become:

$x_{i}^{*}(0)=x_{\mathrm{f}}$

$v^{*}(0)=1$
$T^{*}(0)=1$

$y_{i}^{*}(0)=\frac{\tilde{J}_{i}(0)}{\tilde{J}(0)}$

$u^{*}(0)=0$

$P^{*}(1)=\frac{P_{H}}{P_{1}^{\text {sat }}}$

The input data required to solve the pervaporation model are: (i) feed-side inlet composition, velocity and temperature; (ii) permeate-side temperature and outlet pressure; (iii) membrane element dimensions; (iv) membrane thickness; (v) pure component thermodynamic properties; (vi) solution-diffusion model parameters. Note that the feed-side and permeate-side differential equations involve spatial derivatives only with respect to $x^{*}$ and $y^{*}$, respectively. On the other hand, the component fluxes vary with respect to both $x^{*}$ and $y^{*}$, since the mass transfer rate depends on the feed-side and permeate-side stream conditions. Therefore, numerical solution of the dimensionless model yields predictions of each dependent variable as a function of both the feed and permeate flow directions.

The numerical solution algorithm is inspired by the work of Hickey and Gooding [13]. The key observation is that the feed side is governed by initial value differential equations, while the permeate side is governed by boundary value differential equations. This allows the development of a customized solution algorithm in which numerical integration is used in the feed flow direction and the shooting method is used in the permeate flow direction. The spiral-wound element is discretized in both the $x^{*}$ and $y^{*}$ directions to produce a two-dimensional grid with elements of length $\Delta x^{*}$ and height $\Delta y^{*}$. The number of grid points in the $x^{*}$ and $y^{*}$ directions are denoted $n_{x}+1$ and 
$n_{y}+1$, respectively, where

$\Delta x^{*}=\frac{L}{n_{x}}, \quad \Delta y^{*}=\frac{H}{n_{y}}$

The position on the grid is denoted by the pair $\left(x_{j}^{*}, y_{j}^{*}\right)$, where

$x_{j}^{*}=j \frac{\Delta x^{*}}{L}, \quad 0 \leq j \leq n_{x}$

$y_{j}^{*}=j \frac{\Delta y^{*}}{H}, \quad 0 \leq j \leq n_{y}$

Recall that feed-side and permeate-side variables vary in both the feed and permeate flow directions due to their coupling through the component fluxes. The feed enters the module at $x^{*}=x_{0}^{*}=0$, where the feed-side composition $x_{i}(x, y)$, velocity $v(x, y)$ and temperature $T(x, y)$ are known and constant with respect to $y^{*}$ due to the boundary conditions (61)-(63):

$x_{i}^{*}\left(0, y_{j}^{*}\right)=x_{\mathrm{f}}, \quad v^{*}\left(0, y_{j}^{*}\right)=1, \quad T^{*}\left(0, y_{j}^{*}\right)=1$

The permeate-side velocity at the closed end of the module, where $y^{*}=y_{0}^{*}=0$ is known due to the boundary condition (65): $u^{*}\left(x_{j}^{*}, 0\right)=0$. The permeate-side composition at any point $\left(x_{j}^{*}, 0\right)$ is calculated as follows. By substituting Eq. (60) expressed in terms of molar flux into the boundary condition (64), the following quadratic equation for the permeate-side composition of the first component at the point $\left(x_{j}^{*}, 0\right)$ can be derived:

$a\left[y_{1}^{*}\left(x_{j}^{*}, 0\right)\right]^{2}+b y_{1}^{*}\left(x_{j}^{*}, 0\right)+c=0$

The constants are defined as

$$
\begin{aligned}
a \equiv & P^{*}\left(x_{j}^{*}, 0\right) P_{1}^{\mathrm{sat}}\left(k_{t 1}-k_{t 2}\right), \\
b \equiv & -\left[x_{1}^{*}\left(x_{j}^{*}, 0\right)\left(k_{t 1} P_{1}^{\mathrm{sat}}-k_{t 2} P_{2}^{\mathrm{sat}}\right)\right. \\
& \left.\quad+P^{*}\left(x_{j}^{*}, 0\right) P_{1}^{\mathrm{sat}}\left(k_{t 1}-k_{t 2}\right)+k_{t 2} P_{2}^{\mathrm{sat}}\right], \\
c \equiv & x_{1}^{*}\left(x_{j}^{*}, 0\right) k_{t 1} P_{1}^{\mathrm{sat}}
\end{aligned}
$$

where the subscripts 1 and 2 denote the first component and second component, respectively. Only one solution of the quadratic equation is physically meaningful:

$y_{1}^{*}\left(x_{j}^{*}, 0\right)=\frac{-b-\sqrt{b^{2}-4 a c}}{2 a}$

The feed-side boundary conditions are used to compute the permeate-side variables at the points $\left(0, y_{j}^{*}\right)$ using the shooting method. The overall mass transfer coefficient $k_{\mathrm{t} i}\left(0, y_{j}^{*}\right)$ is computed from the feed-side boundary conditions. An initial guess of the unknown permeate-side pressure at the point $(0,0)$ is generated using the method of Rautenbach and Albrecht [21]. This yields the relation

$$
P_{0}^{*}(0,0) \approx \sqrt{\left[P^{*}(1) P_{1}^{\mathrm{sat}}\right]^{2}+\frac{24 R T_{\mathrm{p}} J(0,0) \mu_{\mathrm{v}} H^{2}}{W^{2}}}
$$

where the flux $J(0,0)$ is estimated from Eq. (60) by replacing $P^{*}(0,0)$ with $P^{*}(1)$. The estimated pressure $P_{0}^{*}(0,0)$ from Eq. (67) and the feed-side composition $x^{*}(0,0)$ are used to compute the component fluxes $J_{i}(0,0)$. Then the three dimensionless permeate-side differential equations are integrated from $y^{*}=0$ to $y^{*}=\Delta y^{*}$ assuming the feed-side and permeate-side properties are approximately constant over this small interval. We use the variable step size integrator ODESSA with a fixed output interval to achieve high accuracy with a reasonably coarse grid. The permeate-side composition $y_{i}^{*}\left(0, \Delta y^{*}\right)$ and pressure $P^{*}\left(0, \Delta y^{*}\right)$ are used to compute the component flux $J_{i}\left(0, \Delta y^{*}\right)$, and the permeate-side differential equations are integrated from $y^{*}=\Delta y^{*}$ to $y^{*}=$ $2 \Delta y^{*}$. This procedure is continued up to the point $(0,1)$ where the permeate collection tube is located. If the difference between the estimated permeate-side pressure at the collection tube and the associated boundary condition satisfies $\left|P^{*}(0,1)-P^{*}(1)\right|>\epsilon$, the estimated permeate pressure at the closed-end of the module is updated as follows:

$P_{k+1}^{*}(0,0)=P_{k}^{*}(0,0)-\beta$

where $P_{k}^{*}(0,0)$ denotes the estimated value of $P^{*}(0,0)$ at the $k$ th iteration of the shooting method, and $\beta$ is a constant value. Albeit somewhat inefficient compare to more sophisticated updating techniques, this simple method leads to convergence because the initial estimated $P_{0}^{*}(0,0)$ given by (67) invariably is too large. The shooting calculation is repeated using the updated permeate pressure value until convergence is achieved. This yields the component fluxes as well as the permeate-side composition, velocity and pressure at the points $\left(0, y_{j}^{*}\right)$.

Using the component fluxes $J_{i}\left(0, y_{j}^{*}\right)$ and the feed-side boundary conditions, the three dimension- 
less feed-side differential equations are integrated from $x^{*}=0$ to $x^{*}=\Delta x^{*}$ assuming the feed-side and permeate-side properties are approximately constant. This yields the feed-side composition, velocity and temperature at the points $\left(\Delta x^{*}, y_{j}^{*}\right)$. The permeate-side velocity and composition at the point $\left(\Delta x^{*}, 0\right)$ are determined as before. The initial guess of the permeate pressure at the closed-end of the module is estimated from the converged solution at the point $(0,0): P^{*}\left(\Delta x^{*}, 0\right) \approx P^{*}(0,0)$. Then the shooting method is used to obtain convergence of the permeate-side variables at the points $\left(\Delta x^{*}, y_{j}^{*}\right)$. These results are used to integrate the feed-side equations from $x^{*}=\Delta x^{*}$ to $x^{*}=2 \Delta x^{*}$. This procedure continues up the points $\left(1, y_{j}^{*}\right)$ where the feed stream exits the module.

\section{Simulation studies}

\subsection{Results}

The proposed model is used to predict the performance of a spiral wound pervaporation module for the separation of binary styrene/ethylbenzene mixtures. The solution-diffusion model parameters derived previously for the polyurethane membrane are used in the simulation studies. Table 5 lists the other model parameters and the nominal operating conditions where $F_{\mathrm{f}}$ is the feed mass flow rate. Note that the two-dimensional grid used for numerical solution of the differential equation model consists of 10,000 points. A single simulation requires approximately 50 min of CPU time on a $550 \mathrm{MHz}$ Pentium III processor. The simulation time can be reduced below $10 \mathrm{~min}$ -

Table 5

Model parameters and nominal operating conditions for simulation studies

\begin{tabular}{llll}
\hline Parameter & Value & Parameter & Value \\
\hline$x_{\mathrm{f}}$ & 0.5 & $L(\mathrm{~cm})$ & 100 \\
$F_{\mathrm{f}}(\mathrm{g} / \mathrm{s})$ & 2000 & $H(\mathrm{~cm})$ & 500 \\
$v_{\mathrm{f}}(\mathrm{cm} / \mathrm{s})$ & 4.52 & $W(\mathrm{~cm})$ & 0.5 \\
$T_{\mathrm{f}}(\mathrm{K})$ & 298.15 & $l(\mu \mathrm{m})$ & 1.0 \\
$T_{\mathrm{p}}(\mathrm{K})$ & 298.15 & $n_{x}$ & 100 \\
$P_{H}(\mathrm{~Pa})$ & 66.65 & $n_{y}$ & 100 \\
$\epsilon$ & $10^{-5}$ & $\beta$ & $10^{-4}$ \\
\hline
\end{tabular}

Table 6

Dependent variables at the four corners of the membrane leaf

\begin{tabular}{|c|c|c|c|c|}
\hline \multirow[t]{2}{*}{ Variable } & \multicolumn{4}{|l|}{ Location } \\
\hline & $(0,0)$ & $(L, 0)$ & $(0, H)$ & $(L, H)$ \\
\hline$x_{1}$ & 0.5 & 0.4955 & 0.5 & 0.4950 \\
\hline$v$ & 4.52 & 4.373 & 4.52 & 4.366 \\
\hline$T$ & 298.15 & 290.48 & 298.15 & 290.39 \\
\hline$y_{1}$ & 0.6740 & 0.6023 & 0.6748 & 0.603 \\
\hline$u$ & 0 & 0 & 3858 & 2291 \\
\hline$P$ & 77.83 & 66.65 & 72.82 & 66.65 \\
\hline
\end{tabular}

utes by increasing the tolerance for the permeate-side shooting calculation to $\epsilon=10^{-4}$.

The feed-side styrene composition, feed-side temperature, permeate-side styrene composition and permeate-side pressure as a function of position along the membrane leaf are shown in Figs. 8-11, respectively. Plots of the feed-side and permeate-side velocities are omitted for the sake of brevity. To facilitate visualization of the three-dimensional graphs, Table 6 contains values of all six dependent variables in physical units at the four corners of the membrane leaf. Most spatial variations are small due to the rather low permselectivity of the polyurethane membrane. As shown in Figs. 8 and 9, the feed-side variables are equal to their boundary conditions at the entrance of the module. The feed-side composition varies in the feed flow $(x)$ direction but changes very little in the permeate flow $(y)$ direction. Similar behavior is observed for the feed-side temperature. Despite the fact that bulk permeate flow is in the $y$-direction, the permeate-side composition is almost constant with respect to $y$ but varies strongly in the $x$-direction. This is attributable to coupling of the feed and permeate sides of the module through the component fluxes. This behavior, which also has been observed by Hickey and Gooding [13], motivates the development of more sophisticated pervaporation models that account explicitly for permeate-side spatial variations in both flow directions. On the other hand, the permeate-side pressure exhibits large variations in the $y$ direction and is essentially constant in the $x$-direction. Note that the pressure is equal to its boundary condition at the location of the permeate collection tube. These simulations demonstrate that the pervaporation model can provide a detailed spatial description of the key feed-side and permeate-side variables. 


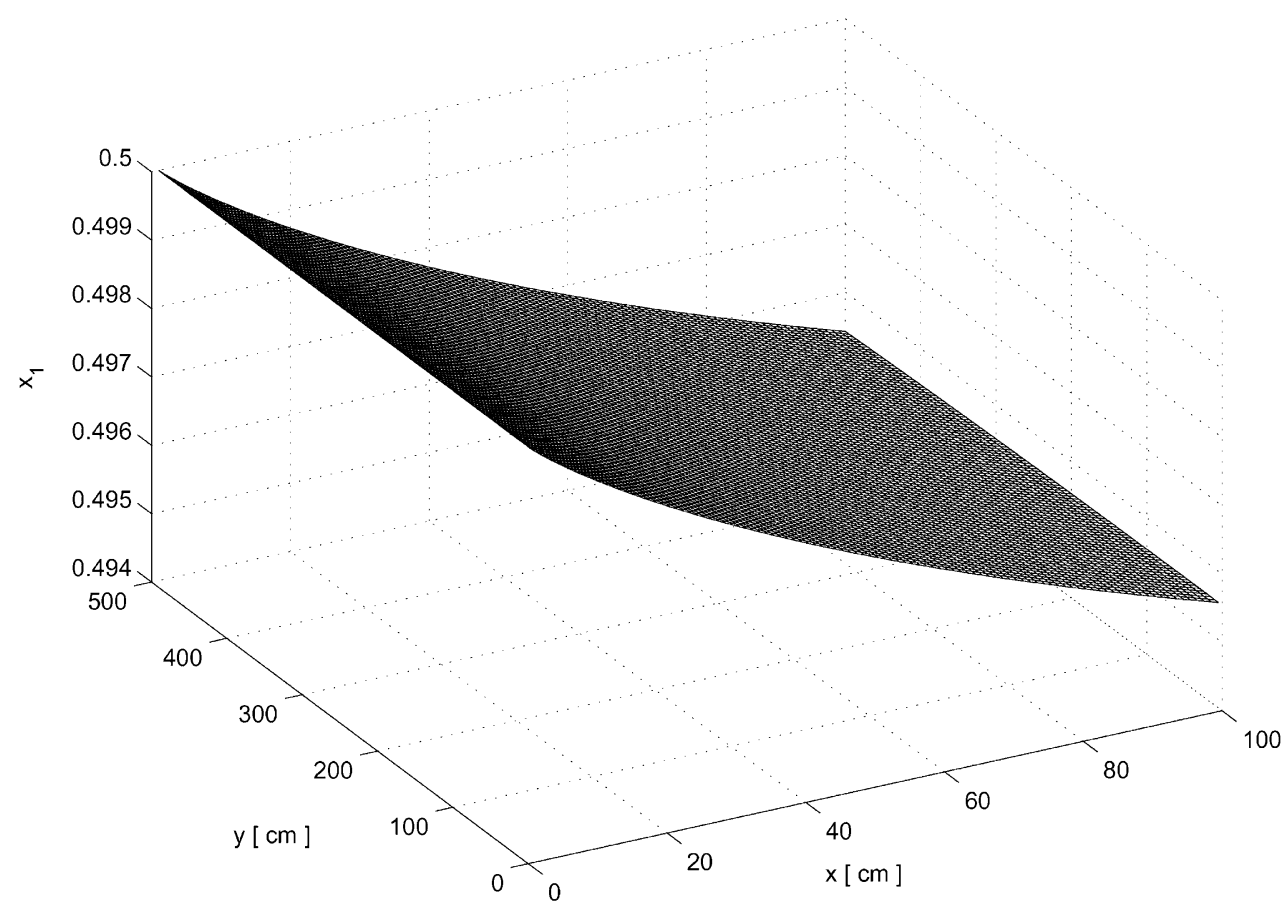

Fig. 8. Feed-side styrene concentration as a function of position.

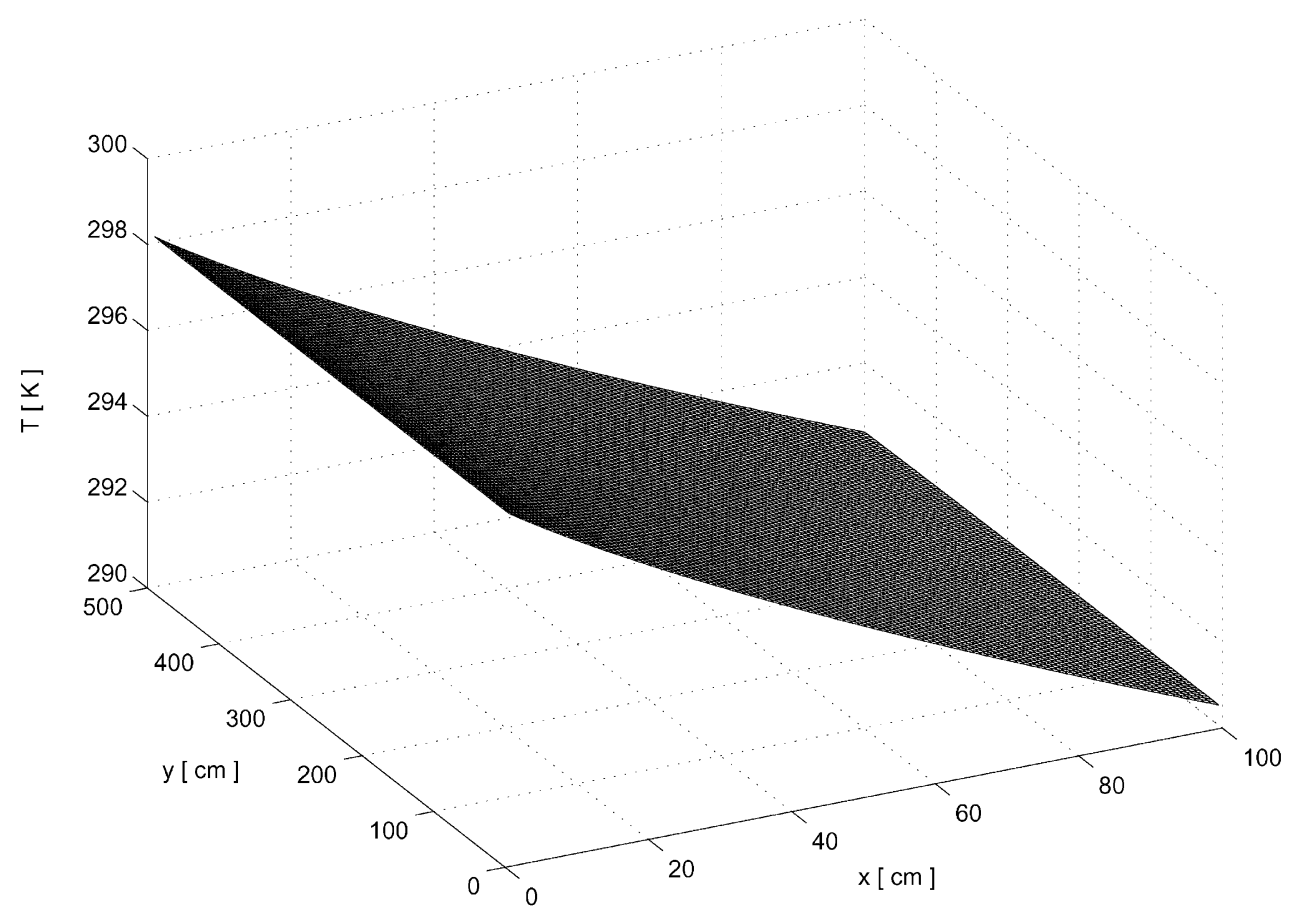

Fig. 9. Feed-side temperature as a function of position. 


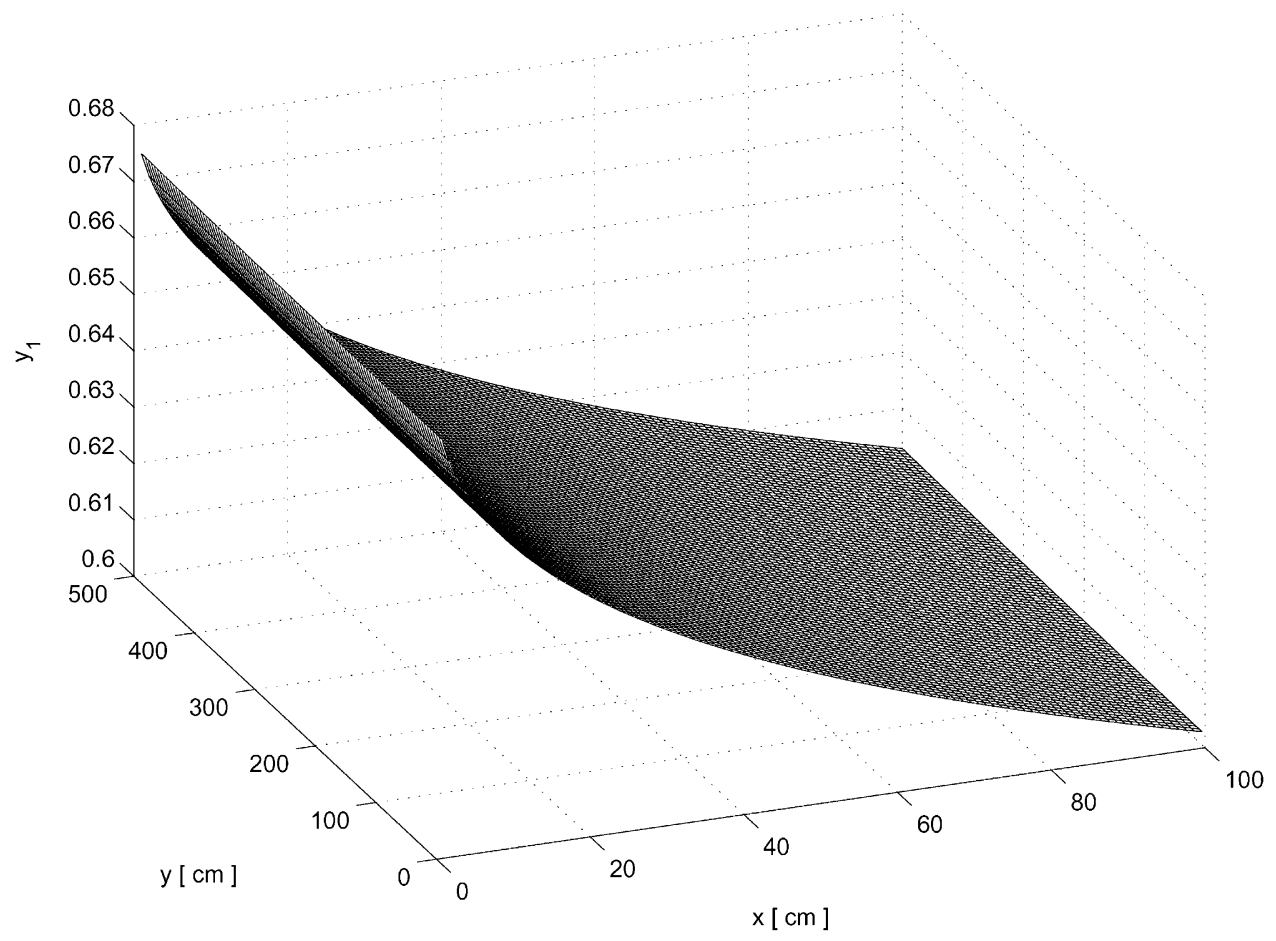

Fig. 10. Permeate-side styrene concentration as a function of position.

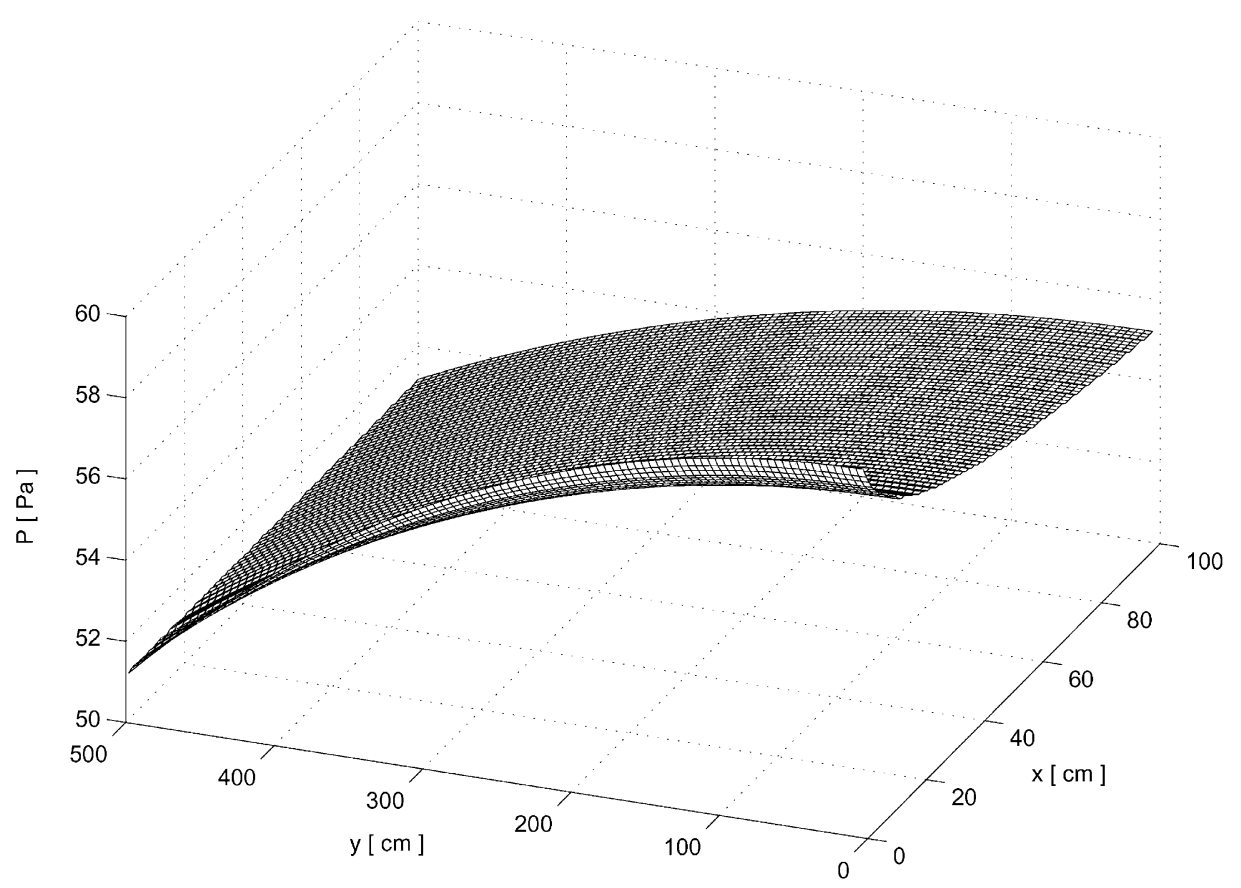

Fig. 11. Permeate-side pressure as a function of position. 


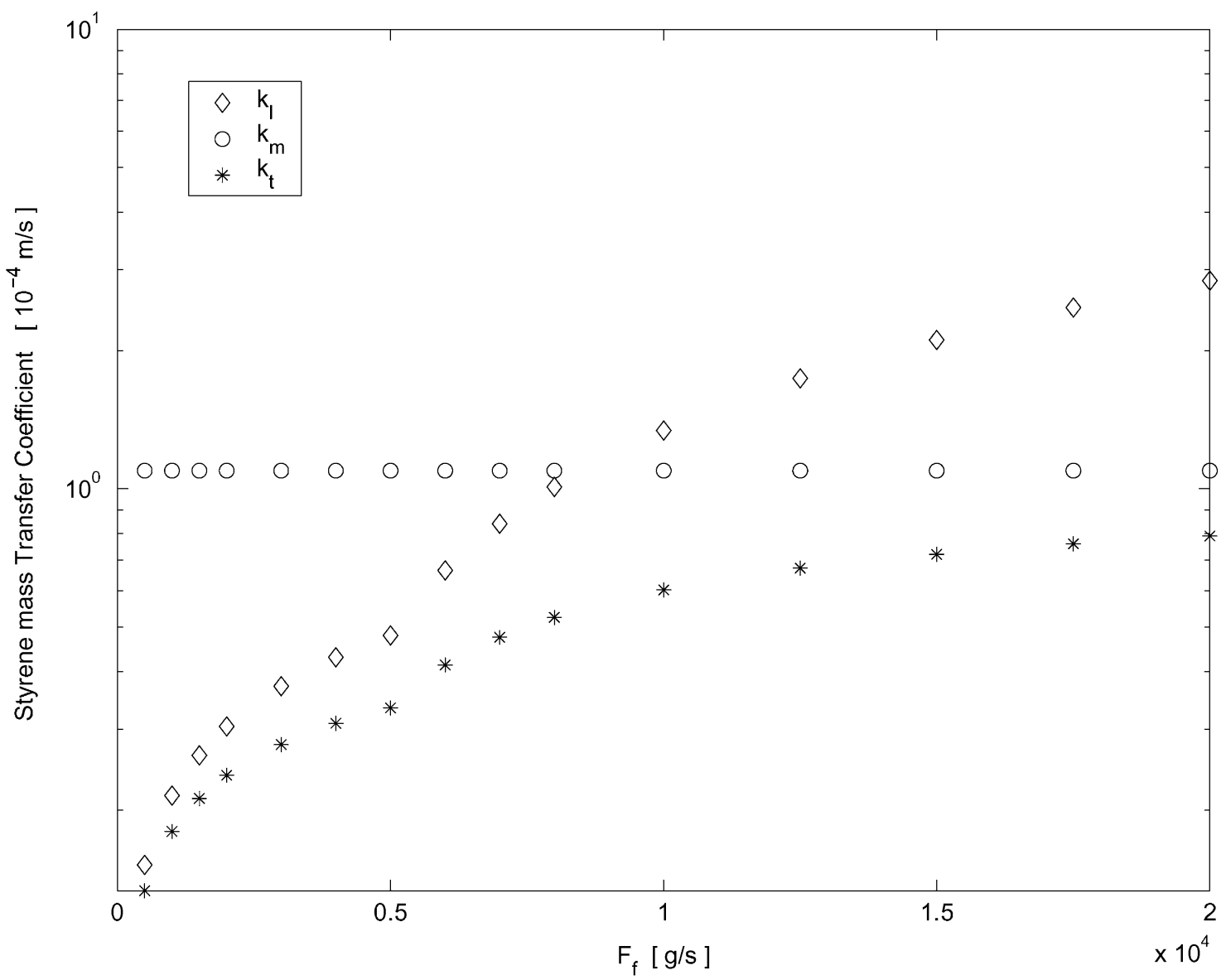

Fig. 12. Styrene mass transfer coefficients as a function of feed flow rate.

Fig. 12 shows the effect of the liquid boundary layer resistance on the overall styrene mass transfer coefficient over a wide range of feed flow rates $\left(F_{\mathrm{f}}\right)$ that cover both laminar and turbulent flow regimes. For simplicity of presentation, the average liquid boundary layer mass transfer coefficient $\hat{k}_{1}$ is used to compute the overall styrene mass transfer coefficient $\left(k_{\mathrm{t} 1}\right)$ and the mass transfer coefficients are expressed in units of $\mathrm{m} / \mathrm{s}$. The membrane styrene mass transfer coefficient $\left(k_{\mathrm{m} 1}\right)$ is essentially constant, while the boundary layer resistance increases with decreasing feed flow rate. The boundary layer resistance is negligible only at very high flow rates where the feed-side Reynolds number is large. Mass transfer is dominated by the membrane resistance under these conditions. At low flow rates, the boundary layer resistance is the dominant effect.

Due to the low permeability of the polyurethane membrane, a rather large membrane area is required to achieve an acceptable flux. Even if the membrane is used for bulk separation in a hybrid pervaporation-distillation process, it is necessary to use multiple modules to achieve the required membrane area. The next set of figures show results for the separation of styrene and ethylbenzene with the 10-module system depicted in Fig. 13. Ten modules are placed in series with the residue stream from the each module serving as the feed stream for the next module. The permeate stream from all the modules are mixed to produce the permeate product. The feed 


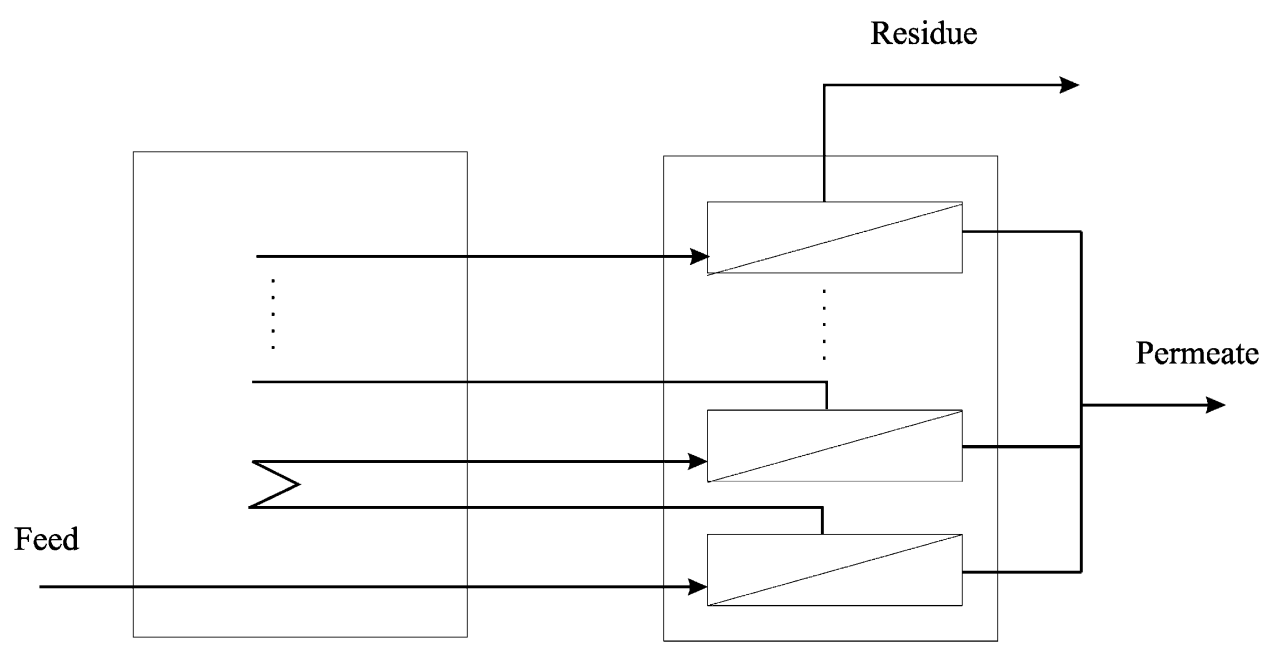

Interstage heating

Fig. 13. Ten modules in series with interstage heating.



Fig. 14. Effect of feed composition on stage cut for 10-module system. 
temperature for each module is increased to the nominal value shown in Table 5 by interstage heating. The separation capability of the system is investigated over a range of feed flow rates and compositions. Feed-side properties are calculated from the residue stream exiting the 10th module, while permeate-side properties are flow weighted averages of the permeate streams from the 10 modules. The properties of interest are the stage cut $\varphi$ and the selectivity $\alpha$ :

$\varphi=\frac{\sum_{k=1}^{10} F_{\mathrm{p} k}}{F_{\mathrm{f}}}, \quad \alpha=\frac{y_{\mathrm{p}}\left(1-x_{\mathrm{f}}\right)}{x_{\mathrm{f}}\left(1-y_{\mathrm{p}}\right)}$

where $F_{\mathrm{p} k}$ is the permeate flow rate of the $k$ th module, and $y_{\mathrm{p}}$ is the styrene composition of the permeate product stream.

The effect of styrene feed composition $\left(x_{\mathrm{f}}\right)$ on the stage cut of the 10-module system is shown in Fig. 14. Because the membrane is styrene selective, the stage cut increases approximately linearly with increasing feed composition. Fig. 15 shows the effect of feed composition on the selectivity. The selectivity decreases rapidly with increasing feed composition for $x_{\mathrm{f}}<0.5$; the decrease is less dramatic for higher $x_{\mathrm{f}}$. Fig. 16 shows the effect of feed flow rate $\left(F_{\mathrm{f}}\right)$ on the stage cut. The stage cut exhibits an exponential decrease with increasing $F_{\mathrm{f}}$. Fig. 17 shows the effect of feed flow rate on the selectivity. The selectivity increases approximately linearly for $F_{\mathrm{f}}>3000 \mathrm{~g} / \mathrm{s}$. For lower flow rates, the selectivity decreases rapidly with decreasing feed flow rate due to the effect of the feed-side liquid boundary layer resistance. These results demonstrate that the pervaporation model can be used to perform process design calculations for non-trivial module configurations.

\subsection{Discussion}

We have developed a differential equation model to simulate the performance of spiral wound membrane

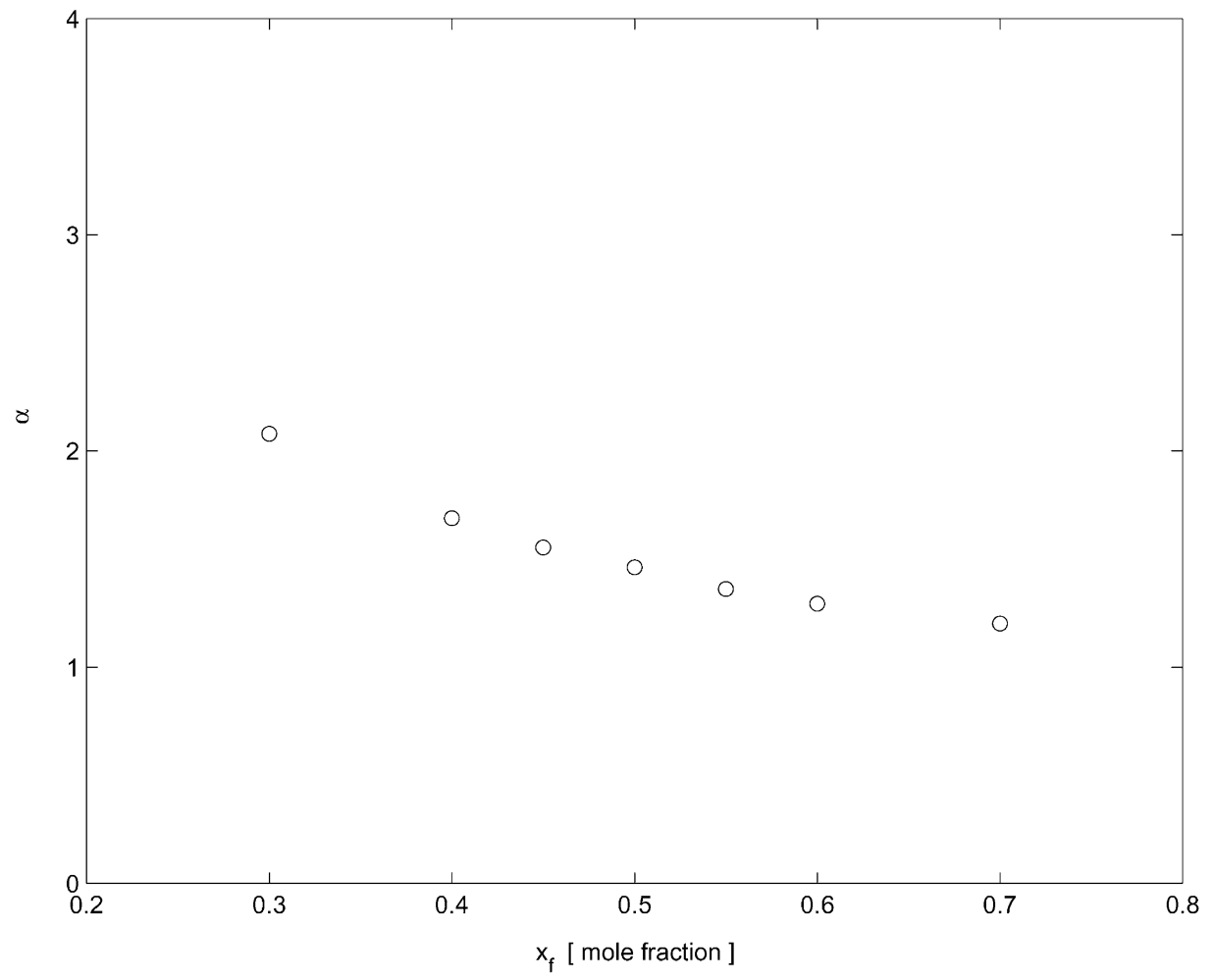

Fig. 15. Effect of feed composition on selectivity for 10-module system. 


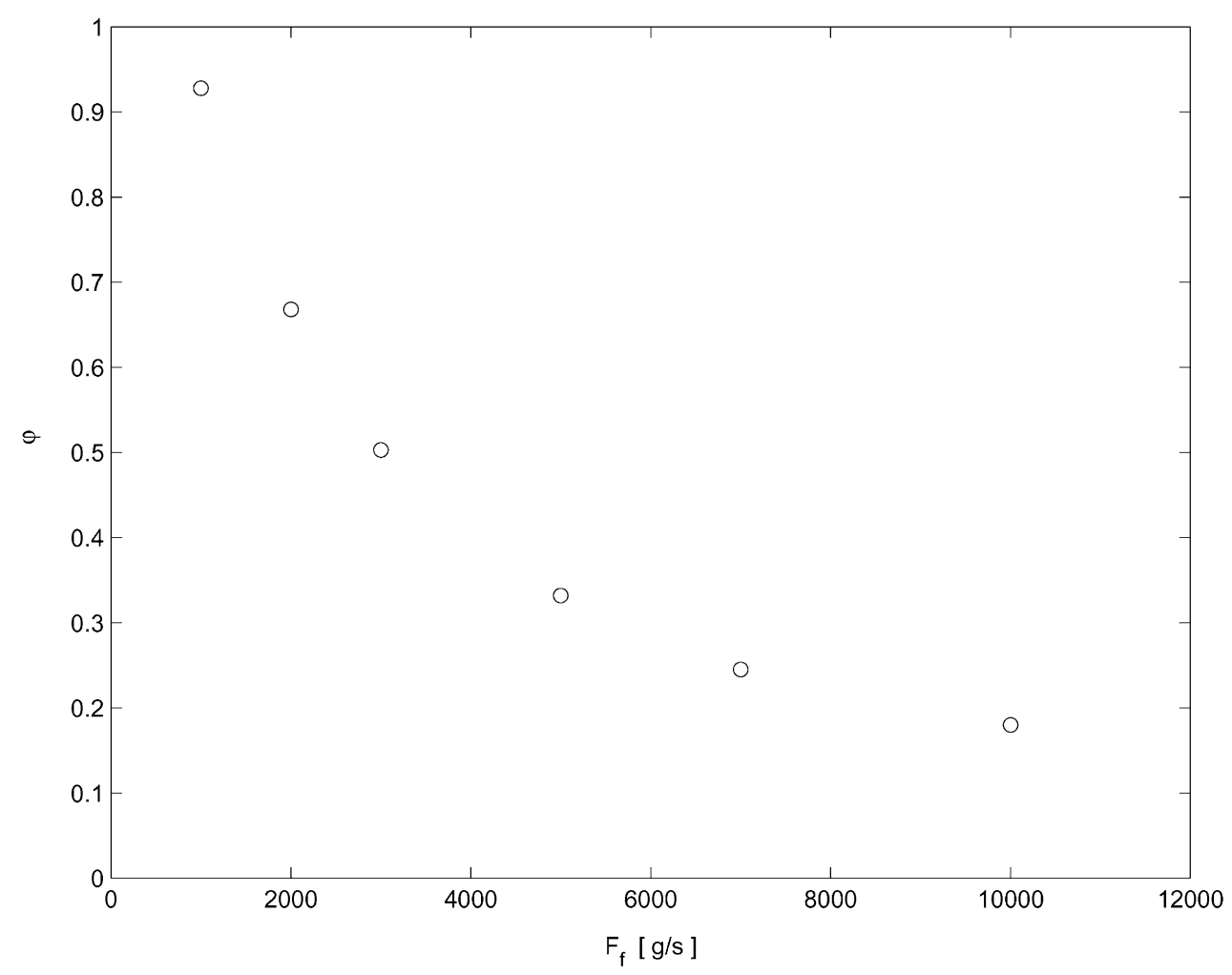

Fig. 16. Effect of feed flow rate on stage cut for 10-module system.

modules for the pervaporative separation of binary liquid mixtures. A detailed solution-diffusion model is utilized to describe permeation through the membrane. Unknown model parameters are determined from sorption and flux data using nonlinear least-squares estimation. The solution-diffusion model is coupled to transport equations for the feed-side composition, velocity and temperature and the permeate-side composition, velocity and pressure. Numerical solution of the model provides predictions of the feed-side and permeate-side variables as a function of position on the two-dimensional membrane leaf. The model has been used to simulate spiral wound pervaporation modules for the separation of styrene and ethylbenzene with a polyurethane membrane. As compared to other pervaporation models available in the literature, the proposed model offers several important advantages for simulating bulk hydrocarbon separations including feed-side balances and a variable mass transfer coefficient.
In addition to providing a general purpose model for spiral wound pervaporation modules, this work is motivated by our interest in hybrid pervaporation-distillation processes for bulk hydrocarbon separations. The proposed model is a useful tool for investigating the economic viability of such hybrid processes. We primarily are interested in hybrid processes for the separation of styrene/ethylbenzene mixtures. As shown in this paper, spiral wound modules constructed from the polyurethane membrane studied in [4] are suitable only for bulk separation due to their modest permselectivity properties. Possible configurations of the hybrid system include processing of the distillation column feed stream, overhead stream, bottom stream or a sidestream by the pervaporation unit. Design studies on hybrid pervaporation-distillation processes for bulk styrene/ethylbenzene separations will be reported in our future publications. 


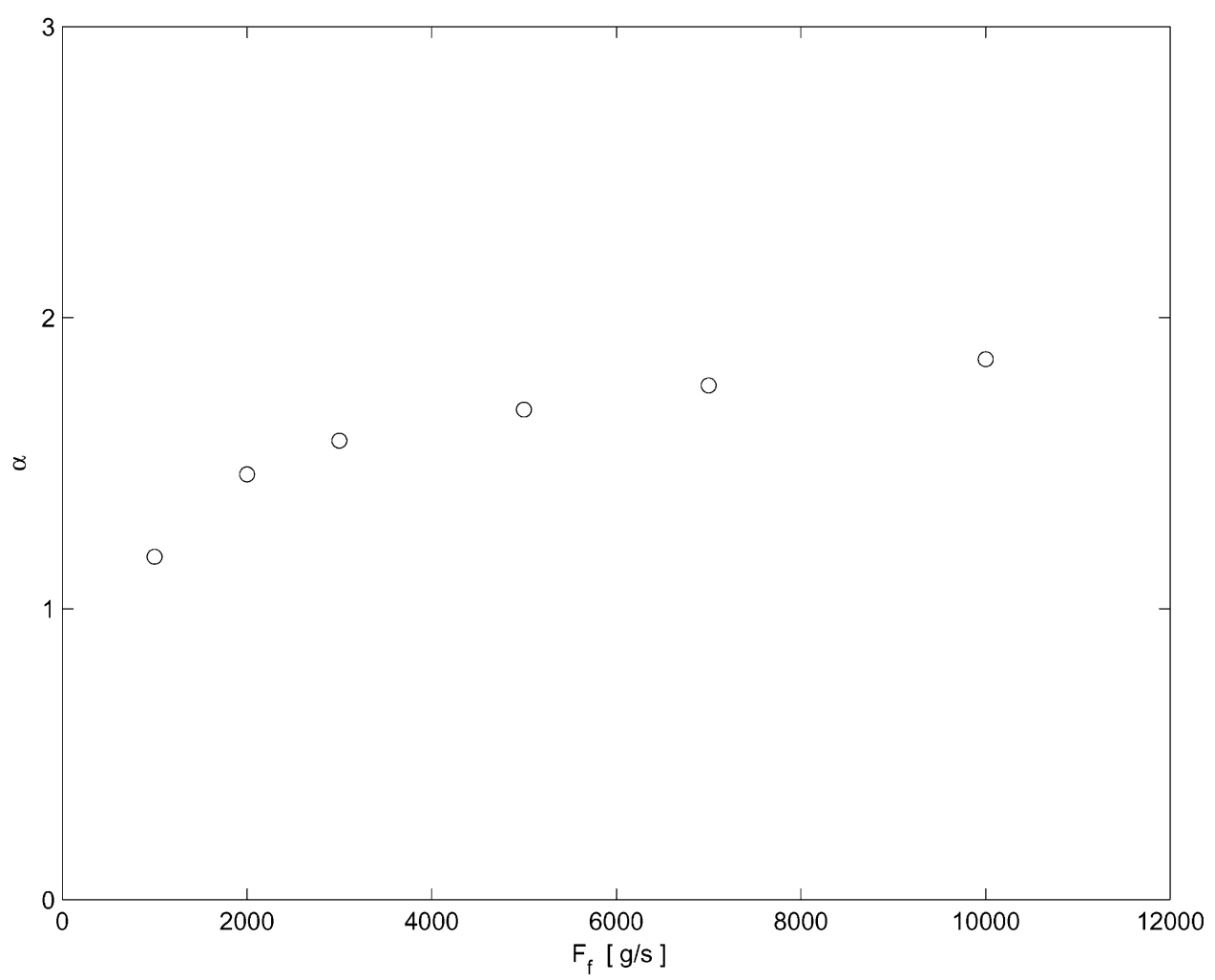

Fig. 17. Effect of feed flow rate on selectivity for 10-module system.

\section{Acknowledgements}

Financial support from the National Science Foundation (Grant CTS-9817298) is gratefully acknowledged. The authors wish to thank Prof. Karsten Thompson (LSU) for his assistance with the lubrication approximation.

\section{Appendix A}

Derivation of the feed-side pressure and velocity equations (38) and (39) begins with the lubrication approximation (37). Because the feed-side pressure $P$ is independent of $z$, integration of Eq. (37) with respect to $z$ yields

$\frac{\partial v_{x}}{\partial z}=\frac{1}{\mu_{1}} \frac{\mathrm{d} P}{\mathrm{~d} x} z+C_{1}$
Let $z=0$ define the centerline of the module with respect to its width. Then the constant of integration $C_{1}=0$, since $\partial v_{x} / \partial z=0$ at $z=0$. Integrating with respect to $z$ a second time yields

$v_{x}(x, z)=\frac{1}{2 \mu_{1}} \frac{\mathrm{d} P}{\mathrm{~d} x} z^{2}+C_{2}$

Let $z_{0}=(1 / 2) W$ denote the location of the membrane surface. The following relation is obtained by evaluating the constant $C_{2}$ using the boundary condition $v_{x}=0$ at $z=z_{0}$ :

$v_{x}=\frac{1}{2 \mu_{1}} \frac{\mathrm{d} P}{\mathrm{~d} x}\left(z^{2}-z_{0}^{2}\right)$

The feed-side volumetric flow rate $Q$ is calculated as

$Q(x)=2 H \int_{0}^{z_{0}} v_{x} \mathrm{~d} z=-\frac{2 H}{3 \mu_{1}} \frac{\mathrm{d} P}{\mathrm{~d} x} z_{0}^{3}$ 
where $H$ is the height of the module. The $z$-averaged velocity $\bar{v}_{x}$ is

$\bar{v}_{x}(x)=\frac{Q}{2 z_{0} H}=-\frac{1}{3 \mu_{1}} \frac{\mathrm{d} P}{\mathrm{~d} x} z_{0}^{2}$

This equation can be rearranged to yield

$\frac{\mathrm{d} P}{\mathrm{~d} x}=-\frac{3 \mu_{1} \bar{v}_{x}}{z_{0}^{2}}$

which is equivalent to Eq. (38), where the average velocity $\bar{v}_{x}(x)$ has been denoted $v(x)$ for simplicity. An equation that relates the velocity $v_{x}$ and the $z$-averaged velocity $\bar{v}_{x}$ is obtained by combining Eqs. (A.1) and (A.2):

$v_{x}=\frac{3}{2} \bar{v}_{x}\left[1-\left(\frac{z}{z_{0}}\right)^{2}\right]$

Because the $y$-component of the feed-side velocity is zero, the continuity equation takes the form [2]

$\frac{\partial v_{x}}{\partial x}+\frac{\partial v_{z}}{\partial z}=0$

This equation is rearranged to yield

$\frac{\partial v_{z}}{\partial z}=-\frac{\partial v_{x}}{\partial x}=-\frac{3}{2} \frac{\mathrm{d} \bar{v}_{x}}{\mathrm{~d} x}\left[1-\left(\frac{z}{z_{0}}\right)^{2}\right]$

Integrating this equation using the boundary condition $v_{z}=0$ at $z=0$ produces

$v_{z}(x, z)=-\frac{3}{2} \frac{\mathrm{d} \bar{v}_{x}}{\mathrm{~d} x}\left(z-\frac{z^{3}}{3 z_{0}^{2}}\right)$

At the membrane surface $z=z_{0}$, the $z$-component of the velocity is equal to the velocity of the permeate stream fluxing through the membrane:

$v_{z}\left(x, z_{0}\right)=-z_{0} \frac{\mathrm{d} \bar{v}_{x}}{\mathrm{~d} x}=\frac{\tilde{J} M_{\mathrm{p}}}{\rho_{\mathrm{p}}}$

where $\tilde{J}$ is the total molar flux, and the molecular weight $M_{\mathrm{p}}$ and density $\rho_{\mathrm{p}}$ of the local permeate are defined by Eqs. (40) and (41), respectively. By setting $z_{0}=(1 / 2) W$ and $\bar{v}_{x}=v$, Eq. (A.3) can be manipulated to yield the differential equation (39) for the average feed-side velocity.

\section{References}

[1] C.O. Bennett, J.E. Myers, Momentum, Heat, and Mass Transfer, McGraw-Hill, New York, NY, 1982.

[2] R.B. Bird, W.E. Stewart, E.N. Lightfoot, Transport Phenomena, Wiley, New York, NY, 1960.

[3] J.P. Brun, C. Larchet, R. Melet, G. Bulvestre, Modeling of the pervaporation of binary mixtures through moderately swelling non-reactive membranes, J. Membr. Sci. 23 (1985) 257.

[4] B. Cao, H. Hinode, T. Kajiuchi, Permeation and separation of styrene/ethylbenzene mixtures through cross-linked poly(hexamethylene sebacate) membranes, J. Membr. Sci. 156 (1999) 43 .

[5] B. Cao, T. Kajiuchi, Pervaporation separation of styrene/ ethylbenzene mixture using poly(hexamethylene sebacate)-based polyurethane membranes, J. Appl. Polym. Sci. 74 (1999) 833.

[6] P. Chaiyavecn, M. Van Winkle, Styrene/ethylbenzene vapor-liquid equilibria at reduced pressures, J. Chem. Eng. Data 4 (1959) 53-59.

[7] J. Crank, The Mathematics of Diffusion, Clarendon Press, Oxford, 1975.

[8] W.H. Deen, Analysis of Transport Phenomena, Oxford, New York, NY, 1998.

[9] X. Feng, R.Y.M. Huang, Liquid separation by membrane pervaporation: a review, Ind. Eng. Chem. Res. 36 (1997) 1048-1066.

[10] B.A. Finlayson, Nonlinear Analysis in Chemical Engineering, McGraw-Hill, New York, 1980.

[11] H.L. Fleming, C.S. Slater, Pervaporation Membrane Handbook, Van Nostrand Reinhold, New York, NY, 1989.

[12] P.J. Flory, Principles of Polymer Chemistry, Cornell University Press, Ithaca, NY, 1953.

[13] P.J. Hickey, C.H. Gooding, Modeling spiral-wound membrane modules for the pervaporation of volatile organic compounds from water, J. Membr. Sci. 88 (1994) 47-68.

[14] J.L. Humphrey, A.F. Seibert, R.A. Koort, Separation technologies: advances and priorities, Technical Report, DOE, 1991.

[15] T. Kataoka, T. Tsuru, S. Nakao, S. Kimura, Permeation equations developed for prediction of membrane performance in pervaporation, vapor permeation and reverse osmosis based on the solution-diffusion model, J. Chem. Eng. Jpn. 24 (1991) 334.

[16] M.H.V. Mulder, T. Franken, C.A. Smolders, Preferential sorption versus preferential permeability in pervaporation, $\mathrm{J}$. Membr. Sci. 22 (1985) 155.

[17] P. Munk, Introduction to Macromolecular Science, Wiley, New York, NY, 1989.

[18] J.M. Prausnitz, R.N. Lichtenthaler, E.G. Azevedo, Molecular Thermodynamics of Fluid-Phase Equilibria, Prentice-Hall, Englewood Cliffs, NJ, 1986.

[19] R. Psaume, P. Aptel, Y. Aurelle, J. Mora, J.L. Bersillon, Pervaporation: importance of concentration polarization in the extraction of trace organics from water, J. Membr. Sci. 36 (1988) 373. 
[20] R. Rautenbach, R. Albrecht, The separation potential of pervaporation. Part 1. Discussion of transport equations and comparison with reverse osmosis, J. Membr. Sci. 25 (1985) $1-23$.

[21] R. Rautenbach, R. Albrecht, The separation potential of pervaporation. Part 2. Process design and economics, J. Membr. Sci. 25 (1985) 25-54.

[22] J. Reale, V.M. Shah, C.R. Bartels, The use of spiral wound modules in pervaporation, in: Proceedings of the 5th
International Conference on Pervaporation Processes in the Chemical Industry, Englewood Cliffs, NJ, 1991, pp. 198-221.

[23] R.C. Reid, T.K. Sherwood, The Properties of Gases and Liquids, McGraw-Hill, 1966.

[24] K.M. Sundaram, H. Sardina, J.M. Fernandezbaujin, J.M. Hildreth, Styrene plant simulation and optimization, Hydrocarb. Proc. 70 (1991) 93-98.

[25] J.G. Wijmans, R.W. Baker, The solution-diffusion model: a review, J. Membr. Sci. 107 (1995) 1. 